\title{
Constraining Lorentz Invariance Violation Using the Crab Pulsar Emission Observed up to TeV Energies by MAGIC
}

\author{
(MAGIC Collaboration),
}

M. L. Ahnen ${ }^{1}$, S. Ansoldi ${ }^{2,3}$, L. A. Antonelli ${ }^{4}$, C. Arcaro ${ }^{5}$, A. Babić ${ }^{6}$, B. Banerjee ${ }^{7}$, P. Bangale $^{8}$, U. Barres de Almeida ${ }^{8}$, J. A. Barrio ${ }^{9}$, J. Becerra González ${ }^{10}$ (1) W. Bednarek ${ }^{11}$ (D) E. Bernardini ${ }^{12,13}$, A. Berti ${ }^{14}$, W. Bhattacharyya ${ }^{12}$, B. Biasuzzi $^{2}$, A. Biland ${ }^{1}$, O. Blanch ${ }^{15}$, S. Bonnefoy ${ }^{9}$, G. Bonnoli ${ }^{16}$, R. Carosi ${ }^{16}$, A. Carosi ${ }^{4}$, A. Chatterjee ${ }^{7}$, S. M. Colak ${ }^{15}$, P. Colin ${ }^{8}$, E. Colombo ${ }^{10}$, J. L. Contreras ${ }^{9}$, J. Cortina ${ }^{15}$, S. Covino ${ }^{4}$ (10, P. Cumani ${ }^{15}$, P. Da Vela ${ }^{16}$, F. Dazzi ${ }^{4}$, A. De Angelis ${ }^{5}$, B. De Lotto ${ }^{2}$, E. de Oña Wilhelmi ${ }^{17}$, F. Di Pierro ${ }^{5}$, M. Doert ${ }^{18}$, A. Domínguez ${ }^{9}$ (), D. Dominis Prester ${ }^{6}$, D. Dorner ${ }^{19}$, M. Doro ${ }^{5}$, S. Einecke ${ }^{18}$, D. Eisenacher Glawion ${ }^{19}$, D. Elsaesser ${ }^{18}$, M. Engelkemeier ${ }^{18}$, V. Fallah Ramazani ${ }^{20}$, A. Fernández-Barral ${ }^{15}$, D. Fidalgo ${ }^{9}$, M. V. Fonseca ${ }^{9}$, L. Font ${ }^{21}$, C. Fruck ${ }^{8}$, D. Galindo ${ }^{22}$, R. J. García López ${ }^{10}$, M. Garczarczyk ${ }^{12}$, D. Garrido ${ }^{21}$, M. Gaug ${ }^{21}$ (1),

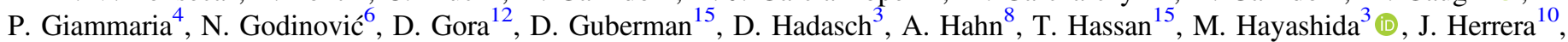

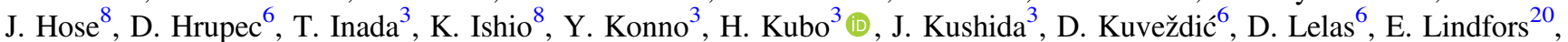
S. Lombardi ${ }^{4}$, F. Longo ${ }^{14}$, M. López ${ }^{9}$, C. Maggio ${ }^{21}$, P. Majumdar ${ }^{7}$, M. Makariev ${ }^{23}$, G. Maneva ${ }^{23}$, M. Manganaro ${ }^{10}$, K. Mannheim ${ }^{19}$, L. Maraschi ${ }^{4}$, M. Mariotti ${ }^{5}$, M. Martínez ${ }^{15}$, D. Mazin ${ }^{3,8}$, U. Menzel $^{8}$, M. Minev ${ }^{23}$, R. Mirzoyan ${ }^{8}$, A. Moralejo ${ }^{15}$, V. Moreno ${ }^{21}$, E. Moretti ${ }^{8}$, V. Neustroev ${ }^{20}$, A. Niedzwiecki ${ }^{11}$ (10, M. Nievas Rosillo ${ }^{9}$ (1), K. Nilsson ${ }^{20}$ (D), D. Ninci ${ }^{15}$, K. Nishijima ${ }^{3}$, K. Noda ${ }^{15}$, L. Nogués ${ }^{15}$, S. Paiano ${ }^{5}$ (1) J. Palacio ${ }^{15}$, D. Paneque ${ }^{8}$, R. Paoletti ${ }^{16}$, J. M. Paredes ${ }^{22}$ (D) , G. Pedaletti ${ }^{12}$, M. Peresano ${ }^{2}$, L. Perri ${ }^{4}$, M. Persic ${ }^{2,4}$, P. G. Prada Moroni ${ }^{24}$, E. Prandini ${ }^{5}$, I. Puljak ${ }^{6}$, J. R. Garcia ${ }^{8}$, I. Reichardt ${ }^{5}$, W. Rhode ${ }^{18}$, M. Ribó ${ }^{22}$ (1),

J. Rico ${ }^{15}$, C. Righi ${ }^{4}$, T. Saito ${ }^{3}$, K. Satalecka ${ }^{12}$, S. Schroeder ${ }^{18}$, T. Schweizer ${ }^{8}$, S. N. Shore ${ }^{24}$, J. Sitarek ${ }^{11}$, I. Šnidarić ${ }^{6}$,

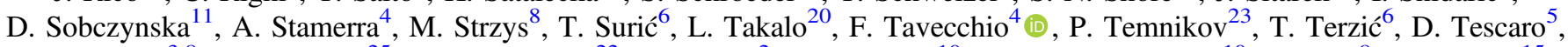

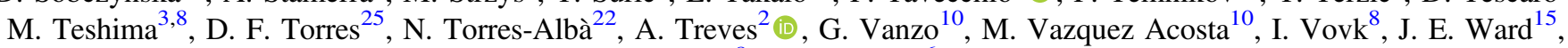
M. Will ${ }^{8}$, and D. Zaric $^{6}$

${ }^{1}$ ETH Zurich, CH-8093 Zurich, Switzerland

${ }^{2}$ Università di Udine, and INFN Trieste, I-33100 Udine, Italy

${ }^{3}$ Japanese MAGIC Consortium: ICRR, The University of Tokyo, 277-8582 Chiba, Japan; Department of Physics, Kyoto University, 606-8502 Kyoto, Japan; Tokai University, 259-1292 Kanagawa, Japan; The University of Tokushima, 770-8502 Tokushima, Japan

${ }^{4}$ INAF-National Institute for Astrophysics, I-00136 Rome, Italy

${ }^{5}$ Università di Padova and INFN, I-35131 Padova, Italy

${ }^{6}$ Croatian MAGIC Consortium: University of Rijeka, 51000 Rijeka, University of Split — FESB, 21000 Split, University of Zagreb — FER, 10000 Zagreb, University of Osijek, 31000 Osijek and Rudjer Boskovic Institute, 10000 Zagreb, Croatia

${ }^{7}$ Saha Institute of Nuclear Physics, HBNI, 1/AF Bidhannagar, Salt Lake, Sector-1, Kolkata 700064, India

${ }^{8}$ Max-Planck-Institut für Physik, D-80805 München, Germany

${ }^{9}$ Universidad Complutense, E-28040 Madrid, Spain

${ }^{10}$ Inst. de Astrofísica de Canarias, E-38200 La Laguna and Universidad de La Laguna, Dpto. Astrofísica, E-38206 La Laguna, Tenerife, Spain

${ }_{11}^{1}$ University of Łódź, Department of Astrophysics, PL-90236 Łódź, Poland

${ }^{12}$ Deutsches Elektronen-Synchrotron (DESY), D-15738 Zeuthen, Germany

${ }^{13}$ Humboldt University of Berlin, Institut für Physik D-12489 Berlin, Germany

${ }^{14}$ University of Trieste and INFN Trieste, I-34127 Trieste, Italy

${ }^{15}$ Institut de Fisica d'Altes Energies (IFAE), The Barcelona Institute of Science and Technology, Campus UAB, E-08193 Bellaterra (Barcelona), Spain ${ }^{16}$ Università di Siena, and INFN Pisa, I-53100 Siena, Italy

${ }^{17}$ Institute for Space Sciences (CSIC/IEEC), E-08193 Barcelona, Spain

${ }_{18}^{18}$ Technische Universität Dortmund, D-44221 Dortmund, Germany

${ }^{19}$ Universität Würzburg, D-97074 Würzburg, Germany

${ }^{20}$ Finnish MAGIC Consortium: Tuorla Observatory and Finnish Centre of Astronomy with ESO (FINCA), University of Turku, Vaisalantie 20, FI-21500 Piikkiö, Astronomy Division, University of Oulu, FIN-90014 University of Oulu, Finland

${ }^{21}$ Unitat de Física de les Radiacions, Departament de Física, and CERES-IEEC, Universitat Autònoma de Barcelona, E-08193 Bellaterra, Spain; markus.gaug@uab.cat ${ }^{22}$ Universitat de Barcelona, ICC, IEEC-UB, E-08028 Barcelona, Spain

${ }^{23}$ Inst. for Nucl. Research and Nucl. Energy, Bulgarian Academy of Sciences, BG-1784 Sofia, Bulgaria

${ }^{24}$ Università di Pisa, and INFN Pisa, I-56126 Pisa, Italy

${ }^{25}$ ICREA and Institute for Space Sciences (CSIC/IEEC), E-08193 Barcelona, Spain

Received 2017 June 21; accepted 2017 July 31; published 2017 September 1

\begin{abstract}
Spontaneous breaking of Lorentz symmetry at energies on the order of the Planck energy or lower is predicted by many quantum gravity theories, implying non-trivial dispersion relations for the photon in vacuum. Consequently, gamma-rays of different energies, emitted simultaneously from astrophysical sources, could accumulate measurable differences in their time of flight until they reach the Earth. Such tests have been carried out in the past using fast variations of gamma-ray flux from pulsars, and more recently from active galactic nuclei and gamma-ray bursts. We present new constraints studying the gamma-ray emission of the galactic Crab Pulsar, recently observed up to $\mathrm{TeV}$ energies by the Major Atmospheric Gamma-ray Imaging Cherenkov (MAGIC) collaboration. A profile likelihood analysis of pulsar events reconstructed for energies above $400 \mathrm{GeV}$ finds no significant variation in arrival time as their energy increases. Ninety-five percent CL limits are obtained on the
\end{abstract}


effective Lorentz invariance violating energy scale at the level of $E_{\mathrm{QG}_{1}}>5.5 \times 10^{17} \mathrm{GeV}\left(4.5 \times 10^{17} \mathrm{GeV}\right)$ for a linear, and $E_{\mathrm{QG}_{2}}>5.9 \times 10^{10} \mathrm{GeV}\left(5.3 \times 10^{10} \mathrm{GeV}\right)$ for a quadratic scenario, for the subluminal and the superluminal cases, respectively. A substantial part of this study is dedicated to calibration of the test statistic, with respect to bias and coverage properties. Moreover, the limits take into account systematic uncertainties, which are found to worsen the statistical limits by about $36 \%-42 \%$. Our constraints would have been much more stringent if the intrinsic pulse shape of the pulsar between $200 \mathrm{GeV}$ and $400 \mathrm{GeV}$ was understood in sufficient detail and allowed inclusion of events well below $400 \mathrm{GeV}$.

Key words: cosmic rays - gamma rays: general - gravitation - methods: data analysis - methods: statistical pulsars: individual (Crab Pulsar)

\section{Introduction}

Common models of quantum gravity (QG) (Rovelli 2004) try to combine Einstein's framework of gravitation with modern quantum field theory, introducing microscopic granular structure and probabilistic dynamics of spacetime. Although none of these scenarios are currently universally accepted, most of them (Kostelecký \& Samuel 1989; Gambini \& Pullin 1999; Douglas \& Nekrasov 2001; Burgess et al. 2002; Magueijo \& Smolin 2002; Hamed-Arkani et al. 2004; Hořava 2009) predict spontaneous violation of the Lorentz invariance (LIV). This can lead to a non-trivial, i.e., energy-dependent dispersion relation of the photon in vacuum and birefringence, as well as an anisotropy of the vacuum. At lower energies, the modified dispersion relation can be parameterized by an effective QG energy scale $\left(E_{\mathrm{QG}}\right)$, which can be on the order of the Planck scale $\left(E_{\mathrm{Pl}}=\sqrt{\hbar c^{5} / G} \approx 1.22 \times 10^{19} \mathrm{GeV}\right)$ or lower. $\mathrm{QG}$ effects are then largely suppressed, but can manifest themselves if photons of different energy travel very large distances and hence accumulate tiny delays that yield potentially measurable effects (Amelino-Camelia \& Smolin 2009).

The group velocity of photons of energy $E \ll E_{\mathrm{QG}}$ can then be parameterized (see, e.g., Amelino-Camelia \& Smolin 2009, Equation (3)) as:

$$
u_{\gamma}(E)=\frac{\partial E}{\partial p} \approx c \cdot\left[1-\sum_{n} \xi_{n} \frac{n+1}{2}\left(\frac{E}{E_{\mathrm{QG}_{n}}}\right)^{n}\right],
$$

where $c$ is the (Lorentz-invariant) speed of light and $\xi_{n}$ is the sign of the change: $\xi_{n}=+1$ for a "subluminal" scenario (decreasing photon speed with increasing energy), $\xi_{n}=-1$ for the "superluminal" case (increasing photon speed with increasing energy), and $\xi_{n}=0$ for the case that the $n$th order is forbidden. The modified dispersion relation can also be written in terms of coupling constants $f_{\gamma}^{(n)}$ of the minimal standard model extensions (SME) (Colladay \& Kostelecký 1998), in which case the substitutions: $n \rightarrow n+2$ and $-f_{\gamma}^{(n)} / E_{\mathrm{Pl}}^{(n-2)} \rightarrow \xi_{n} / E_{\mathrm{QG}_{n}}$ lead to the form chosen in Equation (1) (see also Equations (15) and (74) of Mattingly 2005). Equation (1) neglects terms breaking rotation invariance, which would, however, imply some breaking of boost invariance if they were present (see again Mattingly (2005), chapter 3.1). Terms with $n>0$ produce energy-dependent velocities and are typically considered in time-of-flight experiments. ${ }^{26}$ Because odd terms of $n$ violate CPT (Colladay \& Kostelecký 1998), the $n=2$ term may dominate if CPT is conserved. From a theoretical point of view, subluminal propagation is equally plausible as superluminal (Amelino-Camelia \&

\footnotetext{
${ }^{26}$ Note that terms of $n=-1$ and $n=0$ are also allowed by SME (Colladay \& Kostelecký 1998), but strongly constrained by Earth-based experiments.
}

Smolin 2009); birefringence effects are also possible, in which photons show subluminal and superluminal propagation, depending on their circular polarization state (Kostelecký 2004; Covino \& Gotz 2016). Nevertheless, birefringence has been strongly bound by other means (Gubitosi et al. 2009; Götz et al. 2014; Kislat \& Krawczynski 2017) and will not be considered in the following. Actually, in the framework of an SME approach, current limits from astrophysical polarization measurements constrain variations of the speed of light that are linear with photon energy to several orders of magnitude beyond the Planck scale (Götz et al. 2014; Kislat \& Krawczynski 2017). However, not all quadratic terms, some of which may be realized without vacuum birefringence (Kostelecký \& Mewes 2008), are constrained. Therefore, in the context of possibly constructing CPT conserving theories, constraining the quadratic term $E_{\mathrm{QG}_{2}}$ is now of particular interest.

Exploiting the fast variations of gamma-ray signals from astrophysical sources at cosmological distances to limit LIV was first suggested in Amelino-Camelia et al. (1998). So far, flares from active galactic nuclei (AGNs), exploited first by the Major Atmospheric Gamma-ray Imaging Cherenkov system (MAGIC; Albert et al. 2008) and later by H.E.S.S. (Abramowski et al. 2011), and the very fast flux variations of gamma-ray bursts (GRBs) observed by FERMI (Vasileiou et al. 2013), have boosted sensitivities to such energydependent delays and achieved astonishingly strict limits on $E_{\mathrm{QG}_{1}}$ of well beyond the order of the Planck scale (Vasileiou et al. 2013; Götz et al. 2014). Both types of sources have been detected at cosmological distances, but their maximum observable energy is increasingly limited due to extinction of photons by extra-galactic background light (EBL) (Domínguez \& Ajello 2015). AGN flares, on the other hand, have been observed until energies of several $\mathrm{TeV}$ (Albert et al. 2008; Abramowski et al. 2011), but are closer in distance and show slower rise and fall times than GRBs. Obtained limits on LIV are nevertheless competitive, due to the higher energies achieved, particularly for the quadratic term. Both types of sources require a solid emission model in order to discard any intrinsic, insufficiently understood, energy-dependent effects on the time of emission, which is not yet the case (Bednarek \& Wagner 2008). The effect of this can be mitigated however, through the observation of sources at different redshifts.

Gamma-ray pulsars, albeit being observed many orders of magnitude closer (Abdo et al. 2013) than AGNs or GRBs, have the advantage of precisely timed regular flux oscillations, with periods down to the order of milliseconds, as well as the fact that they are the only stable (in the sense of periodically emitting) candidate sources for astrophysical time-of-flight tests, prescinding from the need of target of opportunity alerts. Sensitivity to LIV can hence be systematically planned and improved using longer observation times. An LIV-induced 
variation of the speed of light would produce a shift in the position of the pulsar peak in its phaseogram, i.e., the emission as a function of the pulsar rotational phase. Moreover, possible intrinsic energy-dependent time delays from the pulsar itself would be observed proportional to its rotational period, while LIV induced effects are not, allowing to disentangle between both, once measurements have been carried out over several years (Otte 2011).

Actually, the first-ever astrophysical limit on LIV was obtained from the Crab Pulsar using optical and radio data (Warner \& Nather 1969). First limits on LIV using gamma-ray emission from the Crab Pulsar were computed from EGRET data up to $2 \mathrm{GeV}$ (Kaaret 1999), and improved by VERITAS using very high energy (VHE) gamma-rays reaching up to $120 \mathrm{GeV}$ (Otte 2011). Recently, the MAGIC collaboration has published the detection of pulsed emission from the Crab Pulsar up to TeV energies (Ansoldi et al. 2016). We exploit this unique set of data to derive improved limits on the effective QG scale using a profile likelihood approach, calibrated both in terms of bias and coverage, and include systematic uncertainties.

This paper is structured as follows. First, we introduce the data set taken on the Crab Pulsar, emphasizing information relevant for LIV searches (Section 2). Second, we perform a basic peak comparison search for signatures of LIV in Section 3, and subsequently construct the full likelihood in Section 4. Several results from applying these methods to data are presented in Section 4.1 and limits to LIV are derived. A thorough calibration of the likelihood using toy Monte Carlo (MC) simulations is performed in Section 4.2, and systematic uncertainties discussed in Section 4.3. The obtained new limits and their implications will be discussed at the end, in Sections 5 and 6 .

\section{The Data Set}

The Crab Pulsar PSR J0534+2200, located at the center of the Crab Nebula in the Taurus constellation, is one of the beststudied pulsars due to its youth, proximity, brightness, and wide spectral coverage (Bühler \& Blandford 2014). It shows a rotation period of $T \approx 33.7 \mathrm{~ms}$, slowly increasing by $\dot{T}=4.2 \times 10^{-13}$. Its distance is still rather poorly determined (Trimble 1973) and generally stated as $2.0 \pm 0.5 \mathrm{kpc}$ (Kaplan et al. 2008). The Crab Pulsar phaseogram (defined as flux — or simply count rate-as a function of the pulsar phase $\phi$ ) shows increased emission in two phase ranges: the main pulse $\mathrm{P} 1$, which has been used to define the zero phase value, and the inter-pulse P2 at $\phi \approx 0.4$. The bridge region between $\mathrm{P} 1$ and $\mathrm{P} 2$ also exhibits emission in optical, X-rays and, as was discovered by MAGIC (Aleksić et al. 2014a), in VHE gamma-rays between 50 and $150 \mathrm{GeV}$. The inter-pulse becomes dominant only at the high end of the spectrum (Abdo et al. 2010a; Aliu et al. 2011; Aleksić et al. 2012a). The energy spectrum of both pulses can be described by simple power-laws from $10 \mathrm{GeV}$ on, and extends to at least $0.5 \mathrm{TeV}$ for $\mathrm{P} 1$ and $1.5 \mathrm{TeV}$ for $\mathrm{P} 2$, as recently measured by MAGIC (Ansoldi et al. 2016).

Contrary to AGNs and GRBs, the gamma-ray signal from the Crab Pulsar at VHE is very background-dominated: generated during a supernova explosion in $1054 \mathrm{AD}$, this young pulsar lies at the center of a strong gamma-ray emitter, the expanding Crab Nebula. The VHE emission of both cannot be spatially resolved so far.

The Major Atmospheric Gamma-ray Imaging Cherenkov system (MAGIC) is located at the Roque de los Muchachos observatory $\left(28^{\circ} .8 \mathrm{~N}, 17^{\circ} .8 \mathrm{~W}, 2200 \mathrm{~m}\right.$ a.s.1.), in the Canary Island of La Palma, Spain. During its first five years of operation, the MAGIC system consisted of a single $17 \mathrm{~m}$ dish telescope (Cortina et al. 2009). In 2009, a second telescope was added with identical structure, but including several major improvements in its reflective surface, camera, and the electronics used for signal processing (Aleksić et al. 2012b).

Between 2011 and 2012, a major upgrade of the MAGIC system was performed to install a new camera and trigger system for MAGIC-I, after which the two telescopes became almost identical in their hardware components (Aleksić et al. 2016a). In stereoscopic observation mode, the system reaches a maximum sensitivity of $\sim 0.6 \%$ of the Crab Nebula flux, for energies above $\sim 300 \mathrm{GeV}$ in $50 \mathrm{hr}$ of observation (Aleksić et al. 2016b). Nevertheless, sensitivity is slightly worse for this source, limited by the strong gamma-ray background from the nebula.

Because the Crab Nebula is the brightest steady source in the VHE gamma-ray sky, it is considered a calibration source for this energy regime and regularly observed for performance tests. The MAGIC telescopes have collected more than $1000 \mathrm{hr}$ of total observation time, taken in every possible hardware configuration during the past 12 years of operation (a detailed summary of the employed data set can be found in Appendix A).

In total, the MAGIC telescopes collected $3080 \pm 460$ excess events from the P2 region, out of which $544 \pm 92$ had reconstructed energies above $400 \mathrm{GeV}$. Moreover, MAGIC was able to confirm that there is a significant difference between the steepness in the spectrum of the Crab main and inter-pulse. We use these very same data to perform tests on LIV, but select only events close to P2 because they will allow us to reach the highest sensitivity, due to the higher reach in energy while keeping the analysis simple enough. For more detailed information about the employed data, we refer the reader to the detection paper (Ansoldi et al. 2016).

\section{Peak Comparison Method}

We first apply a straightforward method that compares the differences in mean fitted pulse positions at different energies (employed in previous LIV searches from Crab Pulsar data (see e.g., Otte 2011)), and later a more sophisticated likelihood approach. Other possible methods, as the so-called PairView or Sharpness-maximization approaches (Vasileiou et al. 2013), have not been exploited in this study.

A simple method to search for energy-dependent delays or advancements in pulse arrival time consists of a direct comparison of peak positions of a pulse.

QG effects predict an average phase delay between photons of mean energies $E_{1}$ and $E_{\mathrm{h}}$ of:

$$
\Delta \phi=\frac{d_{\mathrm{Crab}}}{c P_{\mathrm{Crab}}} \cdot \xi_{n} \frac{n+1}{2} \frac{E_{\mathrm{h}}^{n}-E_{1}^{n}}{E_{\mathrm{QG}_{n}}^{n}},
$$

where $d_{\text {Crab }}$ is the pulsar distance, $c$ the Lorentz-invariant speed of light, $P_{\mathrm{Crab}}$ the pulsar period, and $E_{1}$ and $E_{\mathrm{h}}$ are the mean energies of two separated energy bands, typically chosen to cover the highest part of the observed spectrum and a distinct lower part. 

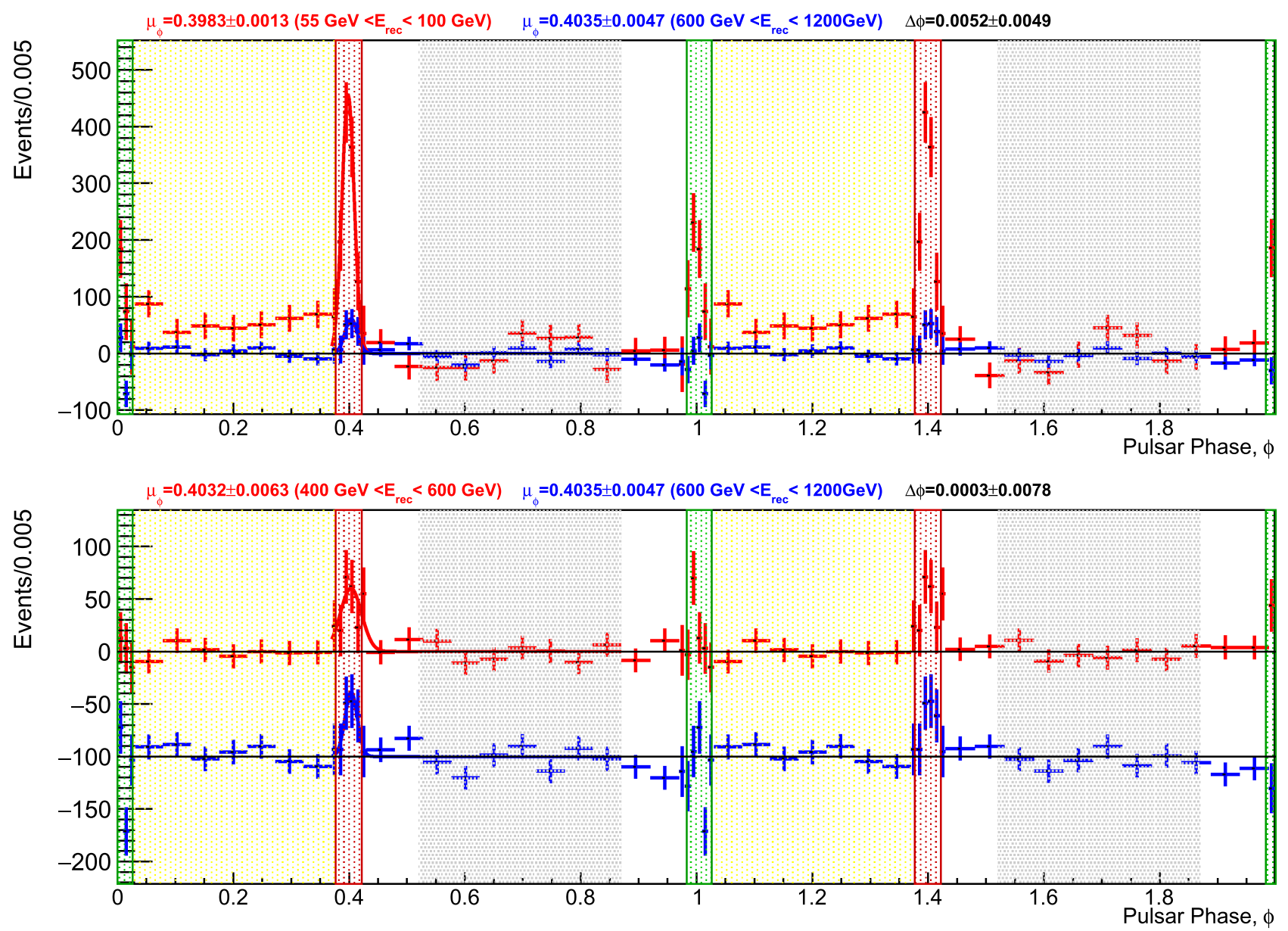

Figure 1. Crab Pulsar folded light curves (two full phases), fitted with a Gaussian pulse shape plus flat background model for the P2 inter-pulse. The main and interpulse regions are binned with a width of 0.005 phases, while the intermediate ranges use coarser bins. The bin width of the pulse regions are chosen to fit a reasonable number of bins into the region, while the coarse bins are selected to fit an integer number of bins into each region. All bin widths have been chosen a priori, independently of the fitting results. The likelihood fit assumes Poissonian fluctuations for the predicted sum of background plus pulse shape from phase 0.37 through 0.87. The background is subtracted here for display only, but is included in the fit. Two distant energy ranges are shown: between 55 and $100 \mathrm{GeV}$ (top) or 400 and $600 \mathrm{GeV}$ (bottom) in red, and above $600 \mathrm{GeV}$ (both figures) in blue. The last one is artificially offset by 100 counts for better visibility. The traditional OFF-region is underlaid with a gray area, the P1 region with green, and the P2 region with red (Ansoldi et al. 2016). The bridge region (Aleksić et al. 2014a) between P1 and P2 is underlaid with yellow.

Limits to $E_{\mathrm{QG}}$ can then be derived from limits on $\Delta \phi$ according to:

$$
E_{\mathrm{QG}_{n}} \gtrsim\left(\xi_{n} \frac{n+1}{2} \frac{d_{\mathrm{Crab}}}{c P_{\mathrm{Crab}}} \frac{E_{\mathrm{h}}^{n}-E_{1}^{n}}{\Delta \phi}\right)^{1 / n}
$$

We use Equation (3) to compare the highest possible energy band with sufficient statistics, i.e., from 600 to $1200 \mathrm{GeV}$, with two lower bands: in one case, they span from the analysis threshold of 55 to $100 \mathrm{GeV}$, while in the second scenario, the lower band limits itself to the data published in Ansoldi et al. (2016) and ranges from 400 to $600 \mathrm{GeV}$. The second choice is motivated by the fact that the detection of pulsar emission at such high energies can hardly be reconciled with the traditional interpretation of pulsar emission through the synchro-curvature process at lower energies. Such a couple of high-energy bands would hence not be affected by a change of the emission mechanism, if such a change happens below $\sim 400 \mathrm{GeV}$, albeit at the price of a worse limit on LIV (see Figure 1).

The mean energies of the selected bands of reconstructed energy, $E_{1} \sim 75 \mathrm{GeV}$ and $465 \mathrm{GeV}$, respectively, for the two low-energy bands, and $E_{\mathrm{h}} \sim 770 \mathrm{GeV}$ for the high-energy one, have been found by MC simulations of the energy spectrum of P2 (Ansoldi et al. 2016), weighted with the correct exposure of the different samples.

The pulses are fitted using the method of maximizing a Poissonian likelihood whose mean is parameterized by one Gaussian over a constant background (see Aleksić et al. 2012a).

A simulated signal of two half-Gaussians of different width, joined at the peak, was also tested, but the $\chi^{2} / \mathrm{NDF}$ of the fits did not improve in any of the tested energy bands. The fit positions $0.3983 \pm 0.0013_{\text {stat. }}$ and $0.4032 \pm 0.0063_{\text {stat. }}$ of the P2 peaks in the phaseogram are obtained for the two lowerenergy bands (compatible with Aleksić et al. 2012a), and $0.4035 \pm 0.0047_{\text {stat. }}$ for the high-energy band, compatible with Ansoldi et al. (2016). A Lorentzian pulse shape is also tested, yielding marginally better $\chi^{2} / \mathrm{NDF}$ and compatible results for the pulse positions. See Table 1 for the obtained results.

The delay in the arrival phase between these two energy ranges is then $\Delta \phi_{\mathrm{P} 2}=0.0052 \pm 0.0049_{\text {stat }} \pm 0.003_{\text {syst }}$ (between $75 \mathrm{GeV}$ and $770 \mathrm{GeV}$ ) and $\Delta \phi_{\mathrm{P} 2}=0.0003 \pm$ $0.0078_{\text {stat }} \pm 0.0030_{\text {syst }}$ (between 465 and $770 \mathrm{GeV}$ ), both compatible with no delay. However, they also show the trend, observed at lower energies, of the mean pulse peak 
Table 1

Obtained Fit Values from the Peak Comparison Method

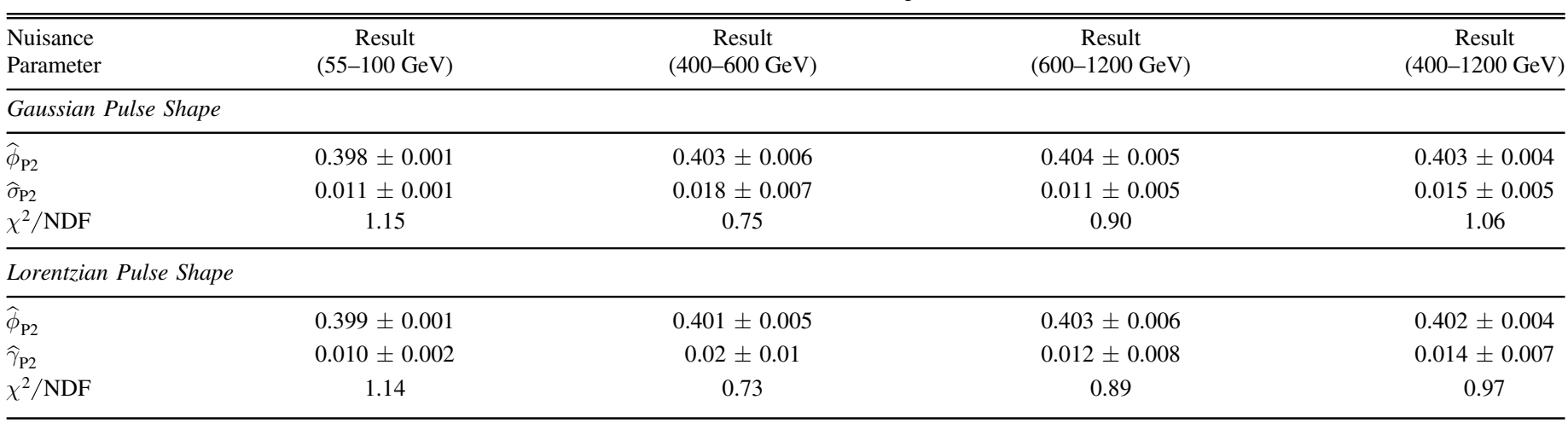

Note. The uncertainties are statistical only. The entries labeled $\phi_{\mathrm{P} 2}$ denote the Gaussian or Lorentzian mean, while $\sigma_{\mathrm{P} 2}$ denotes the Gaussian sigma and $\gamma_{\mathrm{P} 2}$ the Lorentzian half width at half-maximum. The last column shows results for the full range from 400 to $1200 \mathrm{GeV}$; it is not used for the peak comparison, but can be compared to the later results in Table 3.

positions slowly shifting toward higher phases, as their energy increases (see e.g., Abdo et al. 2010a; Aleksić et al. 2012a). The systematic term contains additional uncertainties due to the phase binning and the differences obtained when choosing a Lorentzian light curve model or asymmetric widths (see also Aleksić et al. 2012a). The derived 95\% CL limits on an LIV-induced linear and quadratic phase delay are shown in Table 2.

\section{Maximum Likelihood Method}

More sensitive constraints, which exploit the full information of the MAGIC Crab Pulsar data set, can be obtained with a maximum likelihood (ML) method, first introduced for this kind of search in Martinez \& Errando (2009) and further elaborated in Vasileiou et al. (2013).

We define two new parameters for the linear and quadratic LIV effect intensity, respectively: $\lambda_{1} \equiv 10^{19} \mathrm{GeV} / E_{\mathrm{QG}_{1}}$ and $\lambda_{2} \equiv 10^{12} \mathrm{GeV} / E_{\mathrm{QG}_{2}}$. The mean phase delay produced by the LIV effect under test is then

$$
\Delta \phi_{n}=c_{n} \cdot\left(\lambda_{n} \cdot\left(\frac{E}{\mathrm{GeV}}\right)\right)^{n},
$$

with

$$
\begin{gathered}
c_{1}=\xi_{1} \cdot \frac{d_{\mathrm{Crab}}}{c \cdot P_{\mathrm{Crab}}} \times 10^{-19}\left(\mathrm{GeV}^{-1}\right) \\
c_{2}=\xi_{2} \cdot \frac{3}{2} \frac{d_{\mathrm{Crab}}}{c \cdot P_{\mathrm{Crab}}} \times 10^{-24}\left(\mathrm{GeV}^{-2}\right),
\end{gathered}
$$

such that a positive (negative) value of $\xi$ indicates a subluminal (superluminal) scenario, and a zero intensity of $\lambda_{1,2}$ stands for an infinite LIV energy scale $E_{\mathrm{QG}_{1,2}}$. Note that these definitions differ from those employed by Abramowski et al. (2011) and Vasileiou et al. (2013), particularly for $\lambda_{2}$, which is now directly proportional to $1 / E_{\mathrm{QG}_{2}}$ instead of $1 / E_{\mathrm{QG}_{2}}^{2}$. Being closer to the constrained quantity of interest, particularly $E_{\mathrm{QG}_{2}}$, our definition will allow us to investigate its statistical properties more accurately (see Section 4.2).

Using the profile likelihood ratio method (Murphy \& van der Vaart 2000), we define a test statistic $D_{n}$ for $\lambda_{n}$ of our pulsar data set $\boldsymbol{X}=\left\{E_{i}^{\prime}, \phi_{i}^{\prime}, k_{i}\right\}$, where $E_{i}^{\prime}$ is the reconstructed energy,
Table 2

Obtained 95\%CL Limits from the Peak Comparison Method

\begin{tabular}{lccc}
\hline \hline Case & $\begin{array}{c}55-100 \mathrm{GeV} \\
\text { versus } \\
600-1200 \mathrm{GeV}\end{array}$ & $\begin{array}{c}400-600 \mathrm{GeV} \\
\text { versus } \\
600-1200 \mathrm{GeV}\end{array}$ \\
\hline \multicolumn{4}{c}{$E_{\mathrm{QG}_{1}}(\mathrm{GeV})$} \\
\hline$\xi_{1}=+1$ & $2.5 \times 10^{17}$ & $1.1 \times 10^{17}$ \\
$\xi_{1}=-1$ & $6.7 \times 10^{17}$ & $1.1 \times 10^{17}$ \\
\hline \multicolumn{4}{c}{$E_{\mathrm{QG}_{2}}(\mathrm{GeV})$} \\
\hline$\xi_{2}=+1$ & $1.8 \times 10^{10}$ & & $1.4 \times 10^{10}$ \\
$\xi_{2}=-1$ & $2.9 \times 10^{10}$ & & $1.5 \times 10^{10}$ \\
\hline
\end{tabular}

Note. The first two lines apply to the linear case (delay and advancement), while the last two lines are valid for the quadratic case of LIV (again, delay and advancement). The second column represents the limit on the characteristic LIV energy scale, obtained by comparing the two distant energy bins $55-100 \mathrm{GeV}$ and $600-1200 \mathrm{GeV}$, while the last columns shows the limits obtained from the two adjacent high-energy bins $400-600 \mathrm{GeV}$ and $600-1200 \mathrm{GeV}$.

$\phi_{i}^{\prime}$ is the reconstructed phase, $k_{i}$ is the observation period of event $i$, and $\nu$ is a set of nuisance parameters:

$$
D_{n}\left(\lambda_{n} \mid \boldsymbol{X}\right)=-2 \ln \left(\frac{\mathcal{L}\left(\lambda_{n} ; \widehat{\boldsymbol{\nu}}\left(\lambda_{n}\right) \mid \boldsymbol{X}\right)}{\mathcal{L}\left(\widehat{\lambda}_{n} ; \widehat{\boldsymbol{\nu}} \mid \boldsymbol{X}\right)}\right)
$$

Single-hatted parameters $\left\{\widehat{\lambda}_{n}, \widehat{\nu}\right\}$ maximize the likelihood, while double-hatted parameters $\widehat{\boldsymbol{\nu}}$ are those that maximize $\mathcal{L}$ for a given assumption of $\lambda_{n}$.

Some care needs to be taken for the cases where $\hat{\lambda}_{n}$ comes to lie in an "unphysical" region. We need to define as "unphysical" all those values that cannot be part of a given theory, due to fundamentally different concepts. In our case, this would mean negative values of $\widehat{\lambda}_{n}$ for subluminal theories, i.e., $\xi_{n}>0$ and positive values of $\widehat{\lambda}_{n}$ for superluminal theories, i.e., $\xi_{n}<0$. Following the recommendation of Cowan et al. (2011), we adopt an alternative test statistic that avoids the 
formal use of physical boundaries by construction, namely:

$$
\tilde{D}_{n}\left(\lambda_{n} \mid \boldsymbol{X}\right)= \begin{cases}-2 \ln \left(\frac{\mathcal{L}\left(\lambda_{n} ; \widehat{\mathcal{\nu}}\left(\lambda_{n}\right) \mid X\right)}{\mathcal{L}\left(\lambda_{n} ; \widehat{\boldsymbol{\nu}} \mid \boldsymbol{X}\right)}\right) & \text { if } \operatorname{sgn}\left(\widehat{\lambda}_{n}\right)=\operatorname{sgn}\left(\xi_{n}\right) \\ -2 \ln \left(\frac{\mathcal{L}\left(\lambda_{n} ; \widehat{\widehat{\boldsymbol{\nu}}}\left(\lambda_{n}\right) \mid X\right)}{\mathcal{L}(0 ; \widehat{\boldsymbol{\nu}}(0) \mid \boldsymbol{X})}\right) & \text { otherwise. }\end{cases}
$$

Such a test statistics allows us to set limits on $\lambda_{n}$ at a given confidence level. A one-sided 95\% CL limit is then determined by the value of $\lambda_{n}$ at which $\tilde{D}_{n} \approx 2.705$ (Olive \& Particle Data Group 2014).

We compute $\mathcal{L}$ in the form of an extended likelihood, i.e., the product of the probability density function $(\mathrm{PDF})(\mathcal{P})$ of each event in our data set, considered independent of each other, multiplied by the Poissonian probability to obtain the number of observed events, given the hypothesis $\left\{\lambda_{n} ; \boldsymbol{\nu}\right\}$ (see, e.g., Barlow 1990 and Equation (6) of Kranmer 2015). We fit both $O N$ and $O F F$ regions simultaneously ${ }^{27}$ :

$$
\begin{aligned}
& \mathcal{L}\left(\lambda_{n} ; \boldsymbol{\nu} \mid \boldsymbol{X}\right)=\mathcal{L}\left(\lambda_{n} ; f, \alpha, \phi_{\mathrm{P} 2}, \sigma_{\mathrm{P} 2} \mid\left\{\left\{E_{i}^{\prime}, \phi_{i}^{\prime}\right\}_{i=0}^{N_{k}}\right\}^{N_{s}}\right) \\
= & P(\boldsymbol{\nu}) \cdot \prod_{k=0}^{N_{s}} \exp \left(-g_{k}\left(\lambda_{n} ; \boldsymbol{\nu}\right)-b_{k} \cdot \frac{1+\tau}{\tau}\right) \cdot \prod_{m=0}^{N_{k}^{O F F}} b_{k} . \\
& \quad \prod_{i=0}^{N_{k}^{O N}}\left(g_{k}\left(\lambda_{n} ; \boldsymbol{\nu}\right)+b_{k} / \tau\right) \cdot \mathcal{P}_{k}\left(E_{i}^{\prime}, \phi_{i}^{\prime} \mid \lambda_{n} ; \boldsymbol{\nu}\right),
\end{aligned}
$$

where $g_{k}$ and $N_{k}$ are the expected and observed number of pulsar events of observation period $k$, respectively, with reconstructed energy within a chosen range $\left[E_{\min }^{\prime}, E_{\max }^{\prime}\right]$ (see Section 4.1) and reconstructed phase within the $O N$-phase range $\left[\phi_{\min }^{\prime}, \phi_{\max }^{\prime}\right]$. Note the dependency of $g_{k}$ on both the LIV parameter $\lambda_{n}$ and all nuisance parameters, which are a direct consequence of the limited observed phase range. Similarly, $b_{k}$, which are nuisance parameters, are the corresponding numbers of background events in the standard background control phase $(O F F)$ region [0.52, 0.87] (Fierro et al. 1998), while $\tau$ is the ratio of phase width of the $O F F$, divided by that of the $O N$ region. The phase limits of the $O N$ region have been optimized using simulations (see Section 4.2). The first product runs over $N_{s}$ used observation periods, the second and third over the $N_{k}^{O F F}$ events found in the $O F F$ region, and $N_{k}^{O N}$ events found in the $O N$ regions, respectively, for each observation period $k$. Here, $P(\boldsymbol{\nu})$ is a possible PDF of the nuisance parameters, obtained from external measurements.

A minimum set of nuisance parameters then includes: the P2 flux normalization $f$, its spectral index $\alpha$ (see also Equation (13)), the mean pulse position $\phi_{\mathrm{P} 2}$ and its width $\sigma_{\mathrm{P} 2}$ (see Equation (14)), and the $b_{k}$ background levels. Nuisance parameters may nevertheless also include additional asymmetry parameters, a spectral cutoff, or other variables parameterizing a different pulse model (see later Section 4.1 or 4.3).

The PDF of event $i$ is a normalized combination of PDFs for its measured quantities $\left(E_{i}^{\prime}, \phi_{i}^{\prime}\right)$ to belong either to a pulsar event $S_{k}\left(E_{i}^{\prime}, \phi_{i}^{\prime} \mid \lambda_{n} ; \nu\right)$ or a background event $h_{k}\left(E_{i}^{\prime}\right)$ (see, e.g.,

\footnotetext{
${ }^{27}$ Note that the factors $1 / N_{k}^{O N}$ ! and $1 / N_{k}^{O F F}$ ! have been omitted here because they drop out in the test statistics Equation (8).
}

Aleksić et al. 2014b):

$$
\begin{aligned}
& \mathcal{P}_{k}\left(E_{i}^{\prime}, \phi_{i}^{\prime} \mid \lambda_{n} ; \boldsymbol{\nu}\right) \\
& \quad=\frac{b_{k} / \tau \cdot h_{k}\left(E_{i}^{\prime}\right)+g_{k}\left(\lambda_{n} ; \boldsymbol{\nu}\right) \cdot S_{k}\left(E_{i}^{\prime}, \phi_{i}^{\prime} \mid \lambda_{n} ; \boldsymbol{\nu}\right)}{g_{k}\left(\lambda_{n} ; \boldsymbol{\nu}\right)+b_{k} / \tau},
\end{aligned}
$$

with $h_{k}\left(E^{\prime}\right)$ being the (interpolated) spectral energy distribution of the background, and $S_{k}\left(E_{i}^{\prime}, \phi_{i}^{\prime} \mid \lambda_{n} ; \nu\right)$ the PDF of the pulsar signal for the $k$ th data subsample, respectively. Here, $h_{k}\left(E_{i}^{\prime}\right)$ is a complex combination of cosmic-ray events and gamma-ray images from the Crab Nebula, and is very difficult to model analytically. Because the integral number of background events is always at least a factor of 20 larger than the integrated signal, $\left(b_{k} / \tau\right) / g_{k}>20$, an accurate construction of $h_{k}\left(E_{i}^{\prime}\right)$ is indispensable. We chose to linearly interpolate the binned spectral energy distribution of the background region in double-logarithmic space, and interpolate events without any background events using a linear fit to that distribution. We find that $h_{k}\left(E_{i}^{\prime}\right)$ follows only approximately a power law, showing subtle features such as spectral breaks. We tested different binnings to the original background distribution and found that their effect is acceptable, but nonetheless a non-negligible source of systematic uncertainties (see Section 4.3). The signal PDF, $S_{k}\left(E_{i}^{\prime}, \phi_{i}^{\prime} \mid \lambda_{n} ; \nu\right)$, is calculated as follows:

$$
\begin{aligned}
& S_{k}\left(E_{i}^{\prime}, \phi_{i}^{\prime} \mid \lambda_{n} ; \boldsymbol{\nu}\right) \\
& =\frac{\Delta t_{k} \int_{0}^{\infty} R_{k}\left(E \mid E_{i}^{\prime}\right) \cdot \Gamma_{\mathrm{P} 2}(E, f, \alpha) \cdot F_{\mathrm{P} 2}\left(\phi_{i}^{\prime}, E \mid \lambda_{n} ; \phi_{\mathrm{P} 2}, \sigma_{\mathrm{P} 2}\right) d E}{g_{k}\left(\lambda_{n} ; \boldsymbol{\nu}\right)},
\end{aligned}
$$

where:

1. $\Delta t_{k}$ is the effective observation time for the $k$ th data subsample.

2. $R_{k}$ is the telescope response function of the true photon energy $E$ for the $k$ th subsample, computed as the product of the effective collection area and the energy redistribution function of the instrument. Both have been obtained from Monte-Carlo simulations and fitted to obtain smooth functions in energy.

3. $\Gamma_{\mathrm{P} 2}$ is the pulsar spectrum at $\mathrm{P} 2$, namely:

$$
\begin{aligned}
\Gamma_{\mathrm{P} 2}(E)= & f \cdot\left(E / E_{\mathrm{dec}}\right)^{-\alpha} \cdot \exp \left(-E / E_{b}\right) \\
& \times \mathrm{TeV}^{-1} \mathrm{~cm}^{-2} \mathrm{~s}^{-1} .
\end{aligned}
$$

A previous publication using the same data set (Ansoldi et al. 2016) obtained the values $f_{0}=(5.7 \pm 0.6) \times$ $10^{-10} \mathrm{TeV}^{-1} \mathrm{~cm}^{-2} \mathrm{~s}^{-1}$, a de-correlation energy (Abdo et al. 2010b) $E_{\mathrm{dec}}=50 \mathrm{GeV}$, and $\alpha=(3.0 \pm 0.1)$ in a joint fit with Fermi data, using a pure power law (i.e., $\left.E_{b}:=\infty\right)$. A possible exponential cutoff has only been excluded below $700 \mathrm{GeV}$ so far (Ansoldi et al. 2016). 
4. $F_{\mathrm{P} 2}$ is the pulsar phaseogram model for a given LIV intensity $\lambda_{n}$ and is computed as:

$$
\begin{gathered}
F_{P 2}\left(\phi_{i}^{\prime}, E \mid \lambda_{n} ; \phi_{\mathrm{P} 2}, \sigma_{\mathrm{P} 2}\right)=\int_{0}^{\infty} \frac{1}{2 \pi \sigma_{\mathrm{res}} \sigma_{P 2}^{\prime}} \\
\cdot \exp \left[-\frac{\left(\phi_{i}-\phi_{\mathrm{P} 2}-\Delta \phi\left(E \mid \lambda_{n}\right)\right)^{2}}{2\left(\sigma_{\mathrm{P} 2}^{\prime}\right)^{2}}-\frac{\left(\phi_{i}^{\prime}-\phi\right)^{2}}{2 \sigma_{\mathrm{res}}^{2}}\right] d \phi \\
=\frac{1}{\sqrt{2 \pi} \sigma_{\mathrm{P} 2}} \cdot \exp \left[-\frac{\left(\phi_{i}^{\prime}-\phi_{\mathrm{P} 2}-\Delta \phi\left(E \mid \lambda_{n}\right)\right)^{2}}{2 \sigma_{\mathrm{P} 2}^{2}}\right],
\end{gathered}
$$

where $\sigma_{\mathrm{P} 2}^{\prime}$ is the intrinsic pulse width at the pulsar itself, which may in principle depend on energy, and $\sigma_{\text {res }}$ the instrumental phase resolution, which is dominated by the uncertainties of the pulsar ephemerides, the rms of the timing noise, and the uncertainties of the barycentric corrections. Because the latter contribution is two orders of magnitude smaller than the former (Garrido 2015), the observed width $\sigma_{\mathrm{P} 2}$ can be considered completely dominated by the intrinsic pulse width. Note that the pulse form does not necessarily need to follow a Gaussian, and other, even asymmetric, functions cannot be excluded so far. The effect of different alternative possibilities will be investigated later on (see Section 4.3).

The mean position of the Gaussian includes a signed phase delay produced by the LIV effect under test, described by $\Delta \phi$ (Equation (4)).

5. Here, $g_{k}$ and $b_{k}$ are the normalization constants of $S_{k}$ and $h_{k}$, which depend on the actual realizations of all nuisance parameters, and on $\lambda_{n}$, once these PDFs are integrated within the phase window limits $\phi_{\min }^{\prime}$ and $\phi_{\max }^{\prime}$, and the reconstructed energy limits $E_{\min }^{\prime}$ and $E_{\max }^{\prime}$.

In principle, $\phi_{\min }^{\prime}$ and $\phi_{\max }^{\prime}$ could be chosen to be 0 and 1 , respectively, and the PDF constructed as cyclical. However, in that case, the contributions of P1 and the bridge emission (Aleksić et al. 2014a) need to be modeled as well, unnecessarily complicating the PDF and adding systematic uncertainties to the results. Moreover, it is more computationally efficient to reduce the background as much as possible, by choosing tight windows $\phi_{\min }^{\prime}$ and $\phi_{\max }^{\prime}$ around $\mathrm{P} 2$.

6. The limits for the integration over the true photon energy are formally set to zero and infinity, but physically need to be set to a lower value $E_{\min }$ above which the emission model Equation (13) is considered valid, e.g., a good choice would be the transition from the exponential cutoff to the power-law, around $40 \mathrm{GeV}$. Such a value does not hamper the precision of the overall likelihood, because the minimum reconstructed energy $E^{\prime}$ has been chosen to be $400 \mathrm{GeV}$, sufficiently far from this value in comparison with the energy resolution of $15 \%-20 \%$ (see Appendix A).

7. The choice of the cutoff energy $E_{b}$ is less obvious: the last significant spectral point, obtained with these data, lies at $\sim 1.5 \mathrm{TeV}$ and still fits the power-law (Equation (13)), although an exponential cutoff can only be excluded below $700 \mathrm{GeV}$ at 95\% CL (Ansoldi et al. 2016). A reasonable, justified choice of $E_{b}$ above $700 \mathrm{GeV}$ is hence a priori impossible; its effects on the limits on $\lambda_{n}$ will be studied in Section 4.1.
The PDF for the nuisance parameters flux $(f)$ and spectral index $(\alpha)$ is assumed to be normally distributed and uncorrelated because it was evaluated at the de-correlation energy $E_{\mathrm{dec}}$ (Ansoldi et al. 2016):

$$
P(f, \alpha)=\mathcal{N}\left(\mu_{f}, \sigma_{f}^{2}\right) \cdot \mathcal{N}\left(\mu_{\alpha}, \sigma_{\alpha}^{2}\right),
$$

with $\mu_{f}$ and $\mu_{\alpha}$ being the central fit results for $f_{0}$ and $\alpha$, and $\sigma_{f}$ and $\sigma_{\alpha}$ their statistical uncertainties.

The PDF for the pulse position parameters $\phi_{\mathrm{P} 2}$ and $\sigma_{\mathrm{P} 2}$ had to be assumed flat because no previous information is available about their values, except for this very same data set.

The definition of the likelihood, Equation (11), assumes that the phases have been reconstructed with sufficient precision (we assume the systematic uncertainty in the reconstruction of the phases of the order of $10^{-3}$ in phase), such that any residual uncertainty between reconstructed phase $\phi_{i}^{\prime}$ and true phase $\phi$ can be absorbed in the nuisance parameter $\sigma_{\mathrm{P} 2}$. Similarly, the change of pulsar period from $33.60 \mathrm{~ms}$ in 2007 to $33.69 \mathrm{~ms}$ in 2014 has been absorbed in $\sigma_{\mathrm{P} 2}$. Note that both effects are statistically independent of the reconstructed photon energy.

\subsection{Application of the Profile Likelihood to Data}

The ML algorithm (Equation (8)) is now applied to the MAGIC Crab Pulsar data set (Ansoldi et al. 2016), using $E_{\text {min }}^{\prime}=100 \mathrm{GeV}$ and $E_{\text {min }}^{\prime}=400 \mathrm{GeV}$. Toward even lower energy limits, the background results difficult to model with high accuracy, because gamma-hadron separation works less and less efficiently, especially for those data that were taken with only one telescope. Remember that the analysis leading to this data sample has been optimized for high energies. For the minimization of the profile likelihood, we use the TMINUIT class of ROOT (James \& Roos 1975; Brun \& Rademakers 1996; Brun \& James 2015), employing the MIGRAD, and in case of no success, the SIMPLEX algorithms.

The obtained values of $\lambda_{1,2}$ at the found minima are close to zero in all cases. Table 3 (pulse evolution model 1) shows the obtained nuisance parameters at the minimum. All values obtained for $E_{\min }^{\prime}=400 \mathrm{GeV}$ are compatible with the ones presented in Ansoldi et al. (2016). The results for $E_{\min }^{\prime}=100 \mathrm{GeV}$ are compatible with the numbers presented from previous analyses of data from 40 to $400 \mathrm{GeV}$ (Aliu et al. 2011; Aleksić et al. 2012a). Interestingly, the pulse widths seem to widen (by an about $1 \sigma$ fluctuation into opposite directions) for data below and above $400 \mathrm{GeV}$. This is unexpected, given that a significant shrinking of the pulse width had been observed previously from $\mathrm{GeV}$ energies to beyond $100 \mathrm{GeV}$ (as well as from $\mathrm{MeV}$ to $\mathrm{GeV}$ energies) (Aleksić et al. 2011, 2012a).

For this reason, we test a second pulse evolution model 2, incorporating a linearly changing pulse width with the logarithm of energy (compare also with Figure 3 of Aleksić et al. 2012a):

$$
\sigma_{\mathrm{P} 2}=\sigma_{\mathrm{P} 2,0}-\frac{d \sigma_{\mathrm{P} 2}}{d \log (E)} \cdot \log _{10}\left(E / E_{\min }\right)
$$

Including $d \sigma_{\mathrm{P} 2} / d \log (E)$ in the set of nuisance parameters yields $d \sigma_{\mathrm{P} 2} / d \log (E)=0.00 \pm 0.01$ above $400 \mathrm{GeV}$, compatible with the assumption of a constant pulse width (see Table 3, pulse evolution model 2). For reconstructed energies starting from $100 \mathrm{GeV}$, however, the situation changes and an increasing pulse width is marginally favored, namely: $d \sigma_{\mathrm{P} 2} / d \log (E)=(-6 \pm 4) \times 10^{-3}$. This finding is 
Table 3

Obtained Nuisance Parameter Values at the Minimum for $\lambda_{1,2}$ from the Full Likelihood Method

\begin{tabular}{|c|c|c|}
\hline $\begin{array}{l}\text { Nuisance } \\
\text { parameter }\end{array}$ & $\begin{array}{c}\text { Result } \\
\left(E_{\min }^{\prime}=400 \mathrm{GeV}\right)\end{array}$ & $\begin{array}{c}\text { Result } \\
\left(E_{\min }^{\prime}=100 \mathrm{GeV}\right)\end{array}$ \\
\hline \multicolumn{3}{|l|}{ Pulse evolution model 1} \\
\hline $\begin{array}{l}\widehat{f} \\
\left(\cdot 10^{-10} \mathrm{TeV}^{-1} \mathrm{~cm}^{-2} \mathrm{~s}^{-1}\right)\end{array}$ & $6.3 \pm 0.7$ & $6.2 \pm 0.6$ \\
\hline$\widehat{\alpha}_{\widehat{\phi}_{\mathrm{P} 2}}$ & $\begin{aligned} 2.81 & \pm 0.07 \\
0.403 & \pm 0.003\end{aligned}$ & $\begin{aligned} 2.95 & \pm 0.07 \\
0.401 & \pm 0.001\end{aligned}$ \\
\hline$\widehat{\sigma}_{\mathrm{P} 2}$ & $0.015 \pm 0.003$ & $0.011 \pm 0.002$ \\
\hline \multicolumn{3}{|l|}{ Pulse evolution model 2} \\
\hline $\begin{array}{l}\widehat{f} \\
\left(\cdot 10^{-10} \mathrm{TeV}^{-1} \mathrm{~cm}^{-2} \mathrm{~s}^{-1}\right)\end{array}$ & $6.3 \pm 0.7$ & $5.9 \pm 0.5$ \\
\hline$\widehat{\alpha}$ & $2.81 \pm 0.07$ & $2.92 \pm 0.07$ \\
\hline $\begin{array}{l}\phi_{\mathrm{P} 2} \\
\widehat{\sigma}_{\mathrm{P} 2}\end{array}$ & $\begin{array}{l}0.403 \pm 0.004 \\
0.015 \pm 0.003\end{array}$ & $\begin{array}{l}0.401 \pm 0.001 \\
0.009 \pm 0.002\end{array}$ \\
\hline$\overline{d \sigma_{\mathrm{P} 2} / d \log (E)}$ & $0.00 \pm 0.01$ & $-0.006 \pm 0.004$ \\
\hline \multicolumn{3}{|l|}{ Pulse evolution model 3} \\
\hline $\begin{array}{l}\widehat{f} \\
\left(\cdot 10^{-10} \mathrm{TeV}^{-1} \mathrm{~cm}^{-2} \mathrm{~s}^{-1}\right)\end{array}$ & $\ldots$ & $5.9 \pm 0.6$ \\
\hline$\widehat{\alpha}$ & $\ldots$ & 2.95 (fixed) \\
\hline$\widehat{\phi}_{\mathrm{P} 2}$ & $\ldots$ & $0.4005 \pm 0.0011$ \\
\hline$\overline{\Delta \phi_{\mathrm{P} 2}}$ & $\ldots$ & $0.004 \pm 0.003$ \\
\hline$\widehat{\sigma}_{\mathrm{P} 2,1}$ & $\ldots$ & $0.0089 \pm 0.0009$ \\
\hline$\widehat{\sigma}_{\mathrm{P} 2,2}$ & $\ldots$ & $0.015 \pm 0.003$ \\
\hline$\widehat{E}_{t}(\mathrm{GeV})$ & $\ldots$ & $285 \pm 32$ \\
\hline
\end{tabular}

Note. The uncertainties are statistical only and have been obtained from the diagonal elements of the covariance matrix, provided by MINUIT. Pulse evolution model 1 refers to the original likelihood Equation (8), while pulse evolution models 2 and 3 use the extensions Equations (16) and (17), respectively.

in agreement with the results presented in Ansoldi et al. (2016) and Aleksić et al. (2012a).

Finally, another pulse evolution model 3 , describing an abrupt transition of both pulse position and pulse width, at a fixed (true) energy $E_{t}$, can be tested, namely:

$$
\begin{aligned}
\sigma_{\mathrm{P} 2} & = \begin{cases}\sigma_{\mathrm{P} 2}, 1 & \text { if } E<E_{t} \\
\sigma_{\mathrm{P} 2,2} & \text { if } E \geqslant E_{t}\end{cases} \\
\phi_{\mathrm{P} 2} & = \begin{cases}\phi_{\mathrm{P} 2} & \text { if } E<E_{t} \\
\phi_{\mathrm{P} 2}+\Delta \phi_{\mathrm{P} 2} & \text { if } E \geqslant E_{t} .\end{cases}
\end{aligned}
$$

Just as pulse evolution model 2 contains model 1 in the case of $d \sigma_{\mathrm{P} 2} / d \log (E) \rightarrow 0$, pulse evolution model 3 includes model 1 in the limit $E_{t} \rightarrow \infty$. Figure 2 (left) shows the likelihood at the minimum, when only the parameter $E_{t}$ is varied. One can see that $E_{t} \rightarrow \infty$ is excluded by about $3 \sigma$ significance, if the other nuisance parameters are kept fixed, i.e., at the values obtained below $E_{t}$. Similarly, a transition of the mean pulse position $\left(\Delta \phi_{\mathrm{P} 2}>0\right)$ at $E_{t}=285 \mathrm{GeV}$ is found, albeit with only $1.2 \sigma$ significance. Combining the two individual log-likelihoods for $\sigma_{\mathrm{P} 2,1}$ and $\sigma_{\mathrm{P} 2,2}$ excludes a common pulse width with about $2 \sigma$ significance (see Figure 2 right).

It is evident that more data are required to clearly determine the behavior of the P2 pulse position and width above about
$200 \mathrm{GeV}$, something out of the scope of this paper. However, its influence on the behavior of the profile likelihood below $400 \mathrm{GeV}$ is notable, as can be seen in Figure 3: while the profile likelihood with $E_{\min }^{\prime}=400 \mathrm{GeV}$ appears symmetric around the minimum, the lower energy limit $E_{\min }^{\prime}=100 \mathrm{GeV}$ produces a skewed likelihood with non-standard features, except for the linear case using pulse evolution model 3 . In the quadratic case, a common feature between $\lambda_{2}=20$ and $\lambda_{2}=40$ is observed for all cases, less pronounced for the case of pulse evolution model 3 .

Strikingly, the incorporation of considerably more data between $E^{\prime}=100 \mathrm{GeV}$ and $E^{\prime}=400 \mathrm{GeV}$, which fixes the nuisance parameters $\phi_{\mathrm{P} 2}$ and $\sigma_{\mathrm{P} 2}$ to much more precise values and should consequently produce a steeper profile likelihood, seems to achieve no improvement —or even a worsening —of the precision with which the parameters of interest, $\lambda_{1,2}$, can be determined.

Because we cannot be sure about the correct pulse evolution model for P2, at least below about $300 \mathrm{GeV}$, and to exclude any fake effects on the LIV parameters due to wrongly modeled behavior of the nuisance parameters, we decide to restrict the further LIV-search to the part of the sample starting with $E_{\min }^{\prime}=400 \mathrm{GeV}$. The most probable values are then $\lambda_{1}=-0.4$ and $\lambda_{2}=-1.5$, both statistically compatible with the null hypothesis at the level of $0.1 \sigma$.

\subsection{Calibration of Bias and Coverage}

In this section, the statistical properties bias and coverage of the likelihood Equation (8) are studied with the help of MC simulations. Using the results from the previous section, we perform sets of 1000 simulations of events lists $\left\{E_{i}^{\prime}, \phi_{i}^{\prime}, k_{i} \mid \boldsymbol{\nu}\right\}$. For each simulation set, we randomly sample pulse peak positions, pulse widths, absolute flux levels, and spectral indices of normal distributions centered on the values from Table 3, and widths obtained from a Cholesky decomposition of the covariance matrix (provided by MINUIT from the real data sample), matrixmultiplied with a vector of random normally distributed numbers. With this procedure, the correlations between the nuisance parameters, especially between flux, spectral index, and pulse width, are correctly taken into account (see e.g., Walck 1996; Blobel \& Lohmann 2012). We also test a $\chi^{2}$-distributed pulse width, but obtain results very similar to the normal case.

To simulate the background, parameterized power-laws obtained from the background phase region of real data are used, adapted to each observation period. Phases for the background events are picked from a flat distribution of the entire phase range. Background is hence simulated simultaneously for the signal and background control phase region. The background model is extracted individually from the latter for each simulated data set, according to the algorithm described in the previous section. Because flux and spectral index can vary considerably between each simulation set, the number of reconstructed excess events do so as well, although its mean number coincides with the 544 events (for $E^{\prime}>400 \mathrm{GeV}$ ) presented in Ansoldi et al. (2016).

In order to reduce the computational resources, we consider only events up to $E^{\prime}=7 \mathrm{TeV}$, because an extrapolation of the spectrum predicts on average only one event above that energy. In case of an exponential cutoff $E_{b} \lesssim 7 \mathrm{TeV}$, the prediction would be even smaller. We can hence get rid of a residual background contribution at high energies, to which the likelihood may be sensitive, particularly in the case of quadratic LIV. 
Table 4

Results of the Fits to the Distributions of Reconstructed Values of $\widehat{\lambda}_{1}$ and $\widehat{\lambda}_{2}$

\begin{tabular}{|c|c|c|c|c|c|}
\hline \multirow{2}{*}{ Case } & \multicolumn{2}{|c|}{ Gaussian Fit } & \multicolumn{3}{|c|}{ Student's Fit } \\
\hline & $\sigma$ & $\overline{\chi^{2} / \mathrm{NDF}}$ & $\sigma$ & $\nu$ & $\chi^{2} / \mathrm{NDF}$ \\
\hline & \multicolumn{2}{|c|}{ Linear Model } & & & \\
\hline$E_{\min }^{\prime}=400 \mathrm{GeV}$ no syst. & $7.16 \pm 0.19$ & 2.93 & $8.8 \pm 1.2$ & $2.06 \pm 0.37$ & 0.72 \\
\hline$E_{\min }^{\prime}=400 \mathrm{GeV}$ with syst. & $6.88 \pm 0.20$ & 2.93 & $7.6 \pm 1.1$ & $1.96 \pm 0.39$ & 1.42 \\
\hline$E_{\min }^{\prime}=100 \mathrm{GeV}$ with syst. & $2.26 \pm 0.07$ & 1.10 & $3.70 \pm 0.69$ & $3.58 \pm 0.98$ & 0.44 \\
\hline \multirow[t]{2}{*}{$E_{\min }^{\prime}=400 \mathrm{GeV}$ with syst. CTA } & $1.40 \pm 0.04$ & 1.51 & $2.33 \pm 0.35$ & $3.81 \pm 0.81$ & 0.37 \\
\hline & \multicolumn{2}{|c|}{ Quadratic Model } & & & \\
\hline$E_{\min }^{\prime}=400 \mathrm{GeV}$ no syst. & $9.27 \pm 0.18$ & 3.00 & $8.20 \pm 0.19$ & $2.16 \pm 0.28$ & 1.67 \\
\hline$E_{\min }^{\prime}=400 \mathrm{GeV}$ with syst. & $9.05 \pm 0.14$ & 2.52 & $7.87 \pm 0.21$ & $2.16 \pm 0.31$ & 0.94 \\
\hline$E_{\min }^{\prime}=100 \mathrm{GeV}$ with syst. & $6.79 \pm 0.15$ & 1.86 & $5.99 \pm 0.18$ & $2.93 \pm 0.55$ & 1.12 \\
\hline$E_{\min }^{\prime}=400 \mathrm{GeV}$ with syst. CTA & $2.77 \pm 0.05$ & 5.04 & $2.47 \pm 0.06$ & $1.65 \pm 0.15$ & 1.02 \\
\hline
\end{tabular}

To determine the optimum phase window $\phi_{\min }^{\prime}, \phi_{\max }^{\prime}$, we select \pm 3 standard deviations around the central fit value obtained in Section 3, i.e., $\phi^{\prime} \in[0.3558,0.4495]$, after explicitly checking that a bigger window does not improve the precision of the method. This effectively occurs only when the simulated LIV scale is larger than the limits obtained in Section 3 (for more details, see Garrido 2015).

Stability tests are carried out with simulated data sets of different LIV parameters, the results of which are shown in Figure 4. We find that our algorithm converges correctly on average, under the restriction that a very small, but nevertheless significantly measured, bias of about $4 \%-5 \%$ is present, overestimating the LIV effect. The bias reduces by approximately half when the median of the distribution of $\hat{\lambda}$ is evaluated instead of the mean (not shown in Figure 4). We conclude that the ML estimator is consistent, under the restrictions just mentioned.

In order to demonstrate the statistical behavior of the ML estimator, we simulate and reconstruct the case of no LIV for the following four example cases:

$E_{\min }^{\prime}=400 \mathrm{GeV}$ no systematics: samples with $E^{\prime} \in[400,7000] \mathrm{GeV}$ and effective areas and energy resolution simulated with the same values as those used in the ML analysis.

$E_{\min }^{\prime}=400 \mathrm{GeV}$ with systematics: samples with $E^{\prime} \in[400,7000] \mathrm{GeV}$ and effective areas and energy resolution varied randomly according to the systematic uncertainties stated in Aleksić et al. (2012b, 2016b).

$E_{\min }^{\prime}=100 \mathrm{GeV}$ with systematics: samples with $E^{\prime} \in[100,7000] \mathrm{GeV}$ and effective areas and energy resolution varied randomly according to the systematic uncertainties stated in Aleksić et al. (2012b, 2016b).

$E_{\text {min }}^{\prime}=400 \mathrm{GeV}$ with systematics CTA: samples with $E^{\prime} \in[400,22,000] \mathrm{GeV}$ and effective areas and background multiplied by a factor ten, in order to simulate a toy performance of the Cherenkov Telescope Array (Actis et al. 2011). The energy resolution is estimated from Bernlöhr et al. (2013) and systematic uncertainties according to the CTA calibration requirements (Gaug et al. 2014).

The last case is included to obtain a toy estimate of the sensitivity of this method within a realistic experimental option in the near future for the same amount of observation time, if pulse evolution between 200 and $400 \mathrm{GeV}$ remains insufficiently understood.

Figure 5 shows the obtained distributions of $\hat{\lambda}_{1}$ for these simulated scenarios. In all cases, Gaussian fits yield values of $\chi^{2} / \mathrm{NDF}$ considerably worse than Student's $t$-distributions with argument $t=\widehat{\lambda}_{1} / \sigma \cdot \sqrt{\nu}$, which seem to correctly describe the shape of the distribution, particularly the tails. The addition of systematic uncertainties seem to have an effect on the shape, expressed in Student's $\nu$-parameter, rather than the distribution width $\sigma$. The apparent discrepancy can be understood as an effect of the variations of the two nuisance parameters $\sigma_{\mathrm{P} 2}$ and $\alpha$ (see Appendix B). The slightly non-Gaussian behavior of the reconstructed LIV scales has a direct consequence on the expected coverage properties of the test statistics Equation (8). A cumulative of the Student's distribution with an effective $\nu=(2.06 \pm 0.37) /(1.96 \pm 0.39)$ predicts coverage of only $(93 \pm 2) \% /(92.5 \pm 2) \%$ at $1.64 \sigma$ for a one-sided distribution, instead of the $95 \%$ for the Gaussian case. We check this behavior using pull-plots and find coverages of only $(93.1 \pm 0.6) \% /(91.2 \pm 0.7) \%$, respectively, for the cases of no systematics and included systematics. Confidence limits using the normal values of $1.64 \sigma$ (i.e., $\Delta \tilde{D}_{n}=2.71$ ), will hence be under-covered. In order to retrieve $95 \%$ coverage using a Student's distribution, values of $\delta=1.99 \sigma$ and $\delta=2.11 \sigma$ $\left(\Delta D_{1}=3.96\right.$ and $\left.\Delta D_{1}=4.45\right)$ are predicted instead. A sample with limits extracted using these higher values allow us to retrieve the correct coverage of $95 \%$.

The distributions of $\hat{\lambda}_{2}$ for the simulated scenarios with no LIV are shown in Figure 6. In order to better understand their obviously non-Gaussian shapes, it is instructive to study a simplified version of the likelihood Equation (10). In the hypothetical case of just one observation period, no background, an infinite energy resolution, and infinite phase range, the likelihood can be written as (see also Section 3B of Vasileiou et al. 2013):

$$
\begin{aligned}
\mathcal{L}_{\text {simp }}= & \left(\lambda_{n} \mid \boldsymbol{E}^{\prime}, \boldsymbol{\phi}^{\prime}\right)=\prod_{i=0}^{N_{\text {events }}} \\
& \times \frac{\Delta t_{\mathrm{tot}} \cdot\left\langle A_{\mathrm{eff}}\left(E_{i}^{\prime}\right)\right\rangle \cdot \Gamma_{\mathrm{P} 2}\left(E_{i}^{\prime}\right) \cdot F_{\mathrm{P} 2}\left(\phi_{i}^{\prime}, E_{i}^{\prime} \mid \lambda_{n}\right)}{g} .
\end{aligned}
$$



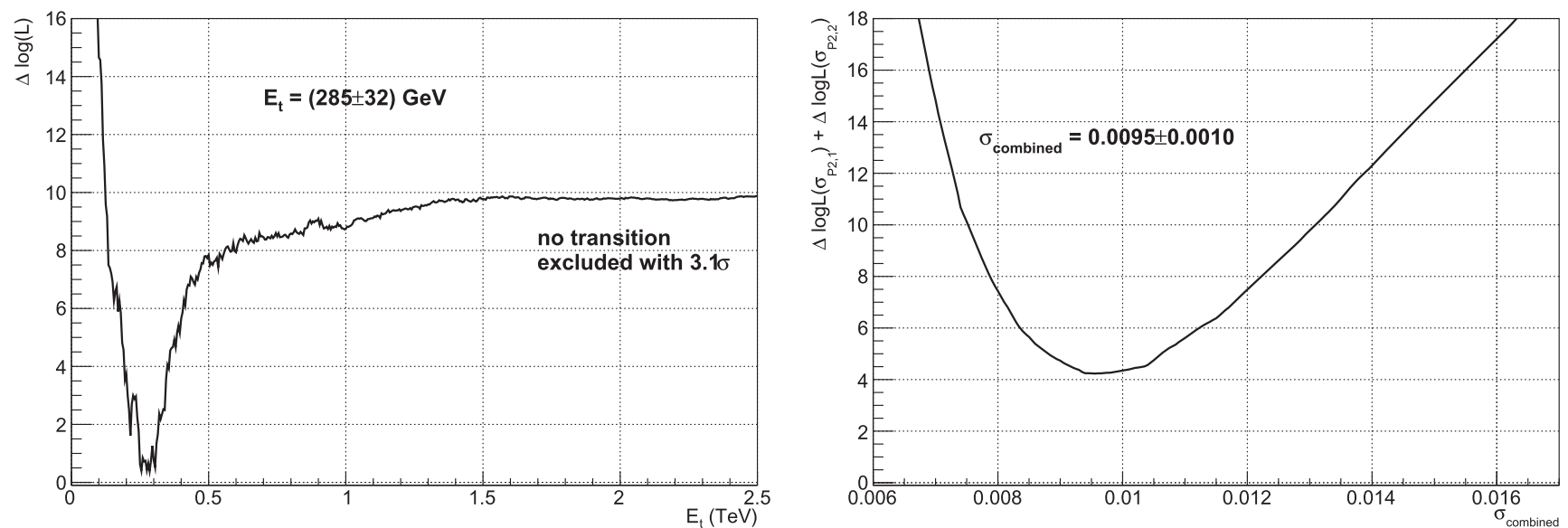

Figure 2. Left: $\log$-likelihood ratio (Equation (8)) as a function of the transition parameter $E_{t}$, under the assumption of no LIV ( $\lambda_{1}=0$ ). Right: the combined loglikelihoods for $\sigma_{\mathrm{P} 2,1}$ and $\sigma_{\mathrm{P} 2,2}$, with the other nuisance parameters kept fixed at the minimum in both cases.

For this simplified likelihood, the expectation value for $\widehat{\lambda}_{n}$ can be calculated analytically, yielding:

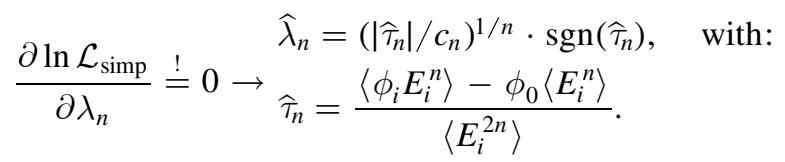

If the phases $\phi_{i}$ are now distributed normally, so will be the values of $\widehat{\pi}_{1} \sim \mathcal{N}\left(\mu_{\hat{\pi}}, \sigma_{\widehat{\pi}_{1}}^{2}\right)$, but not $\widehat{\lambda}_{2}$, because of the squareroot involved. Instead, the PDF of $\widehat{\lambda}_{2}$ has the form ${ }^{28}$

$$
\begin{aligned}
\mathcal{P}_{\text {simp }}\left(\widehat{\lambda}_{2}\right)= & \frac{2}{\sqrt{2 \pi}\left(\sigma_{\widehat{\jmath}_{2}} / c_{2}\right)} \\
& \cdot \exp \left(-\frac{\left(\widehat{\lambda}_{2} \cdot\left|\widehat{\lambda}_{2}\right|-\left(\mu_{\widehat{\lambda}_{2}} / c_{2}\right)\right)^{2}}{2\left(\sigma_{\widehat{\tau}_{2}} / c_{2}\right)^{2}}\right) \cdot\left|\widehat{\lambda}_{2}\right|,
\end{aligned}
$$

Alternatively, if $\widehat{\tau}_{n}$ is distributed according to a Student's $t$-distribution, the PDF for $\widehat{\lambda}_{2}$ yields:

$$
\begin{aligned}
\mathcal{P}_{\text {stud }}\left(\widehat{\lambda}_{2}\right)= & \frac{2}{\sqrt{\pi}\left(\sigma_{\widehat{\jmath}_{2}} / c_{2}\right)} \cdot \frac{\Gamma((\nu+1) / 2)}{\Gamma(\nu / 2)} \\
& \cdot\left(1+\frac{\left(\widehat{\lambda}_{2} \cdot\left|\widehat{\lambda}_{2}\right|-\left(\mu_{\widehat{\tau}_{2}} / c_{2}\right)\right)^{2}}{\left(\sigma_{\widehat{\imath}_{2}} / c_{2}\right)^{2}}\right)^{-(\nu+1) / 2} \cdot\left|\widehat{\lambda}_{2}\right| .
\end{aligned}
$$

Equation (19) is, strictly speaking, the PDF of the square of a normally distributed variable, while Equation (20) is the PDF of the square of a variable distributed according to Student's $t$-distribution. ${ }^{29}$

Contrary to the linear case, the distribution Equation (19) predicts slight over-coverage, while the Student's of 2nd-order predicts marginal under-coverage of $(94.5 \pm 1) \%$, which is found back in the data, namely $(94.2 \pm 0.5) \%$.

\footnotetext{
${ }^{28}$ Following the rule that if $\boldsymbol{X}$ is a random variable that is distributed like $f_{X}(\boldsymbol{X})$, then a variable transformation $y=g(X)$ yields $f_{Y}(y)=f_{x}\left(g^{-1}(y)\right)$. $\frac{d}{d y} g^{-1}(y)$.

${ }^{29}$ For large values of $\mu_{\widehat{\tau}_{2}} / \sigma_{\widehat{\tau}_{2}}$, both distributions converge to a Gaussian. Substituting $\tilde{\mu}=\sqrt{\mid \mu_{\widehat{\tau}_{2} \mid} / c_{2}} \cdot \operatorname{sgn}\left(\mu_{\widehat{\tau}_{2}}\right)$ and $\tilde{\sigma}=\sqrt{\sigma_{\widehat{\tau}_{2}} / c_{2}}$, or for the Student's case, $\tilde{\sigma}=\sqrt{\sigma_{\widehat{\tau}_{2}} /\left(c_{2} \cdot \sqrt{\nu}\right)}$, the variance of Equation (19) can be derived as $V\left[\widehat{\lambda}_{2, \text { simp }}\right]=\sqrt{2 / \pi} \cdot \tilde{\sigma}^{2}$ for the case of $\tilde{\mu}=0$, and for the Student's case Equation (20) $V\left[\widehat{\lambda}_{2, \text { stud }}\right]=2 / \sqrt{\pi \nu} \cdot \Gamma((\nu+1) / 2) / \Gamma(\nu / 2) \cdot \nu /(\nu-1) \cdot \tilde{\sigma}^{2}$, which converges against the Gaussian variance for $\nu \rightarrow \infty$.
}

In order to retrieve 95\% coverage using a Student's distribution, values of $\delta=1.66 \sigma\left(\Delta D_{2}=2.76\right)$ are predicted.

Figure 7 displays predicted $p$-values and suggested new confidence intervals from different integrated one-sided probability distributions: a normal distribution, both of the statistical parameter $x$ as its square $x^{2}$ and Student's $t$-distributions parameterized with the $\nu$-values obtained from the fits to the distributions of reconstructed LIV parameters from the simulations (Figures 5 and 6).

Finally, we calculate limits from each of the simulated samples for the previously found test statistic differences $\tilde{D}_{n}\left(\lambda_{n}^{95 \% \mathrm{CL}}\right)=\Delta \tilde{D}_{n}$. Figure 8 shows the resulting limits.

\subsection{Systematic Uncertainties}

A change of the binning for the spectral energy distribution of the background within reasonable ranges affects the obtained limits by $\leqslant 10 \%$.

As seen in the previous section, inclusion of the systematic uncertainties in reconstructed energy and effective area requires evaluating the test statistic at a larger increase, $\Delta D_{1}=4.45$ instead of $\Delta D_{1}=3.96$, leading to a $\sim 6-7 \%$ increase in the upper limit for the linear case, while no effect has been found for the quadratic case. From the obtained statistical precision of the parameters to fit Equation (20), we can estimate that their effect must be smaller than 5\% (95\% CL) on the limit to $\lambda_{2}$.

The effect of possible inter-pulse shapes differing from a standard Gaussian is more complicated to assess because, in principle, a panoply of different shapes is possible. Until a better theoretical understanding of the pulse shape is also available, we test two easily implemented alternatives: a Lorentzian-shaped pulse, of the form

$$
N=\left(\sigma_{L} / \pi\right) /\left(\left(\phi-\phi_{\mathrm{P} 2}\right)^{2}+\sigma_{L}^{2}\right)
$$

and an asymmetric Gaussian shape.

Evaluating the test statistic on real data using the Lorentzian pulse shape model Equation (21) instead of the Gaussian Equation (14), the limits on $\lambda_{1}$ change by maximal $6 \%$, while those on $\lambda_{2}$ improve by up to $14 \%$.

We address the possibility of asymmetric pulse shapes, as usually observed at lower energies (Abdo et al. 2010a), using a possible extension of the Gaussian pulse shape with an 

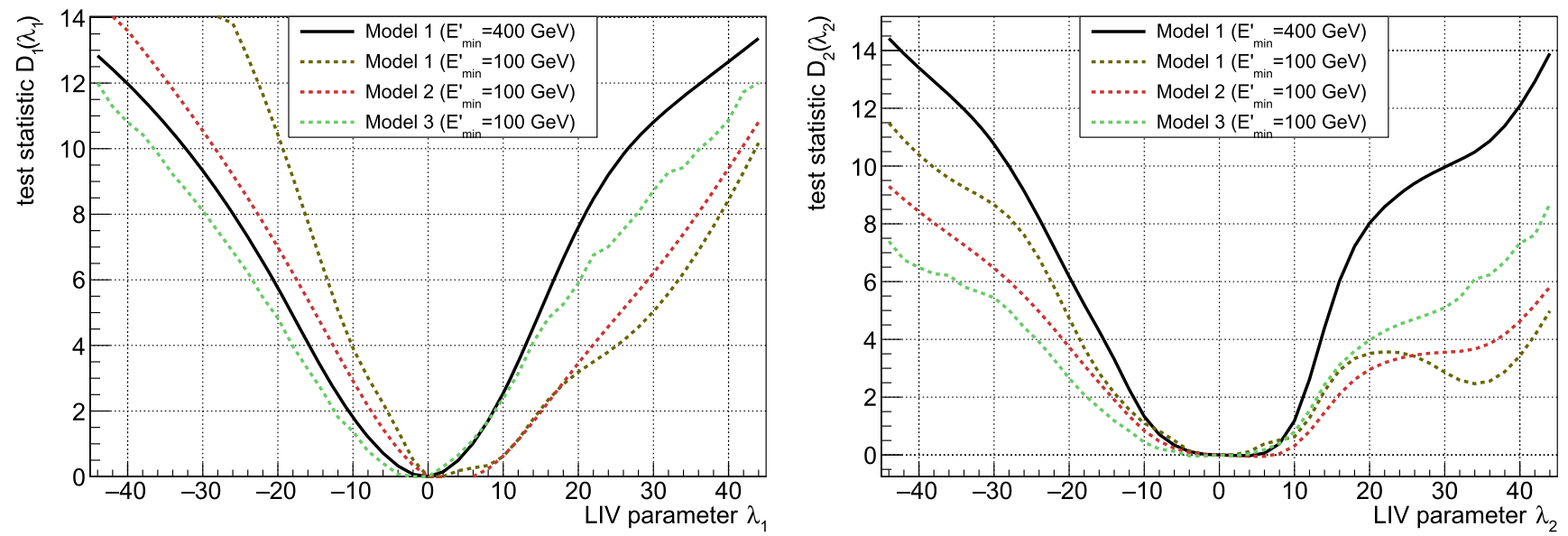

Figure 3. Test statistic (Equation (8)) as a function of the linear LIV parameter $\lambda_{1}$ (left) and the quadratic LIV parameter $\lambda_{2}$ (right). The different pulse evolution models (model 1, model 2, and model 3) have been used, as have two lower energy limits (see text for details).

asymmetry parameter $\Delta \sigma_{\mathrm{P} 2}^{\prime}$ :

$$
\begin{aligned}
& F_{\mathrm{P} 2}^{\text {asym }}\left(\phi_{i}^{\prime}, E \mid \lambda_{n} ; \phi_{\mathrm{P} 2}, \sigma_{\mathrm{P} 2}\right) \\
& =\left\{\begin{array}{c}
\sqrt{\frac{2}{\pi}} \cdot \frac{1}{2 \cdot \sigma_{\mathrm{P} 2}^{\prime}+\Delta \sigma_{\mathrm{P} 2}^{\prime}} \cdot \exp \left[-\frac{\left(\phi_{i}^{\prime}-\phi_{\mathrm{P} 2}-\Delta \phi\left(E \mid \lambda_{n}\right)\right)^{2}}{2\left(\sigma_{\mathrm{P} 2}^{\prime}\right)^{2}}\right] \\
\text { for: } \quad \phi_{i}^{\prime} \geqslant \phi_{\mathrm{P} 2}+\Delta \phi\left(E \mid \lambda_{n}\right) \\
\sqrt{\frac{2}{\pi}} \cdot \frac{1}{2 \cdot \sigma_{\mathrm{P} 2}^{\prime}+\Delta \sigma_{\mathrm{P} 2}^{\prime}} \cdot \exp \left[-\frac{\left(\phi_{i}^{\prime}-\phi_{\mathrm{P} 2}-\Delta \phi\left(E \mid \lambda_{n}\right)\right)^{2}}{2\left(\sigma_{\mathrm{P} 2}^{\prime}+\Delta \sigma_{\mathrm{P} 2}^{\prime}\right)^{2}}\right] \\
\text { for: } \quad \phi_{i}^{\prime}<\phi_{\mathrm{P} 2}+\Delta \phi\left(E \mid \lambda_{n}\right) .
\end{array}\right.
\end{aligned}
$$

A replacement of the pulse shape model $F_{\mathrm{P} 2}$ (Equation (14)) by $F_{\mathrm{P} 2}^{\mathrm{asym}}$ and addition of $\Delta \sigma_{\mathrm{P} 2}^{\prime}$ to the list of nuisance parameters yields $\Delta \sigma_{\mathrm{P} 2}^{\prime}=0.0137_{-0.0032}^{+0.0041}$, with the hypothesis of a symmetric pulse excluded by $2.4 \sigma$ (see Figure 9). The obtained limits on $\lambda_{n}$ improve by at least $\sim 30 \%$ when including the possibility of asymmetric pulse shapes. However, note that the asymmetry parameter and $\lambda$ are anti-correlated: $\rho\left(\lambda_{1}, \Delta \sigma_{\mathrm{P} 2}^{\prime}\right)=-0.47, \rho\left(\lambda_{2}, \Delta \sigma_{\mathrm{P} 2}^{\prime}\right)=-0.16$, and such an improvement is hence expected. However, until a pulse shape asymmetry is significantly established by more data, we refrain from using it to improve the limits on LIV.

An exponential cutoff in the energy spectrum at energy $E_{b}$ (see Equation (13)) at the currently published constraint of $700 \mathrm{GeV}$ (Ansoldi et al. 2016) worsens the limits on $\lambda_{1,2}$ by almost a factor of four. We add $E_{b}$ to the list of nuisance parameters and find no cutoff as the most probable hypothesis, as stated in Ansoldi et al. (2016). Assuming no LIV, and profiling the rest of nuisance parameters with respect to $E_{b}$, we obtain a new limit of $4.3 \mathrm{TeV}(95 \% \mathrm{CL})$ on the cutoff, and the previous limit of $700 \mathrm{GeV}$ disfavored from the most probable hypothesis of no cutoff with a $p$-value of $5 \times 10^{-4}$. See Appendix $C$ for further details. Evaluating the limits on LIV at $E_{b}=4.3 \mathrm{TeV}$, we observe a worsening on the order of $30 \%$ for both linear and quadratic LIV.

Contributions of the bridge emission leaking into the inter-pulse region have not been taken into account in the construction of the likelihood. The flux of the bridge from $\phi=$ $(0.026-0.377)$ has been measured to $\Gamma_{\text {bridge }}(E)=(12.2 \pm 3.3) \cdot$ $(E / 100 \mathrm{GeV})^{-(3.35 \pm 0.79)} \times 10^{-11} \mathrm{TeV}^{-1} \mathrm{~cm}^{-2} \mathrm{~s}^{-1} \quad$ (Aleksić et al. 2014a). Any homogeneous coverage of P2 by the bridge would have no effect on the LIV analysis, but in the worst case, the bridge emission leaks into parts of P2, distorting the pulse shape in an energy-dependent way. If we conservatively assume no spectral cutoff, such a contribution of the bridge to half the P2 pulse might amount to $10 \%$ of the observed excess events on average. We introduce such a case into our toyMC simulation and obtain a worsening of the limits of less than $5 \%$.

The (un-modeled) slow-down of the pulsar frequency, possible glitches of pulsar phase, and mainly the uncertainties of the currently available measurements of the distance to the Crab Pulsar all contribute to the astrophysical uncertainties.

Table 5 summarizes the systematic uncertainties studied. Assuming they are all un-correlated, they add up quadratically to about $\lesssim 42 \%$ for the linear and $\lesssim 36 \%$ for the quadratic case.

A different class of systematic uncertainties relates to intrinsic energy-dependent pulse position drifts from the pulsar itself. Such intrinsic delays may superimpose a possible LIV effect, and in the worst case, mimic or cancel part of its signature. We do not include these in Table 5 and the derived limits, but will briefly discuss them here.

Generally, the origin of VHE pulsar emission up to $\mathrm{TeV}$ energies is still debated in the literature (Aharonian et al. 2012; Du et al. 2012; Hirotani 2013), albeit it is out of the question that inverse Compton scattering must be at play in some way or another (Hirotani 2001; Aleksić et al. 2011; Lyutikov et al. 2012; Bogovalov 2014). Energy-dependent time drifts of the pulse can then follow those of the illuminating electron population, or the illuminated seed UV and X-ray photons, although this is less plausibly because of the involved Klein-Nishina scattering regime. A time dependency of the mean scattering angle is also possible.

At lower (seed photon) energies, dependencies of the pulse peak positions have previously been studied in detail in the literature (Mineo et al. 1997; Massaro et al. 2006, 2000), particularly throughout the strong X-ray signal regime. These studies find constancy of the peak positions of both P1 and P2 throughout more than three orders of magnitude, albeit the pulse widths and shapes do change throughout the X-ray domain. $^{30}$

To make numerical predictions of the size of a possible LIVmimicking effect is clearly beyond the scope of this paper. We

\footnotetext{
${ }^{30}$ Note, e.g., that the phase boundaries, and particularly $f_{3}$ remain constant, both in Massaro et al. (2000) and the more complex multicomponent fits of Massaro et al. (2006).
} 

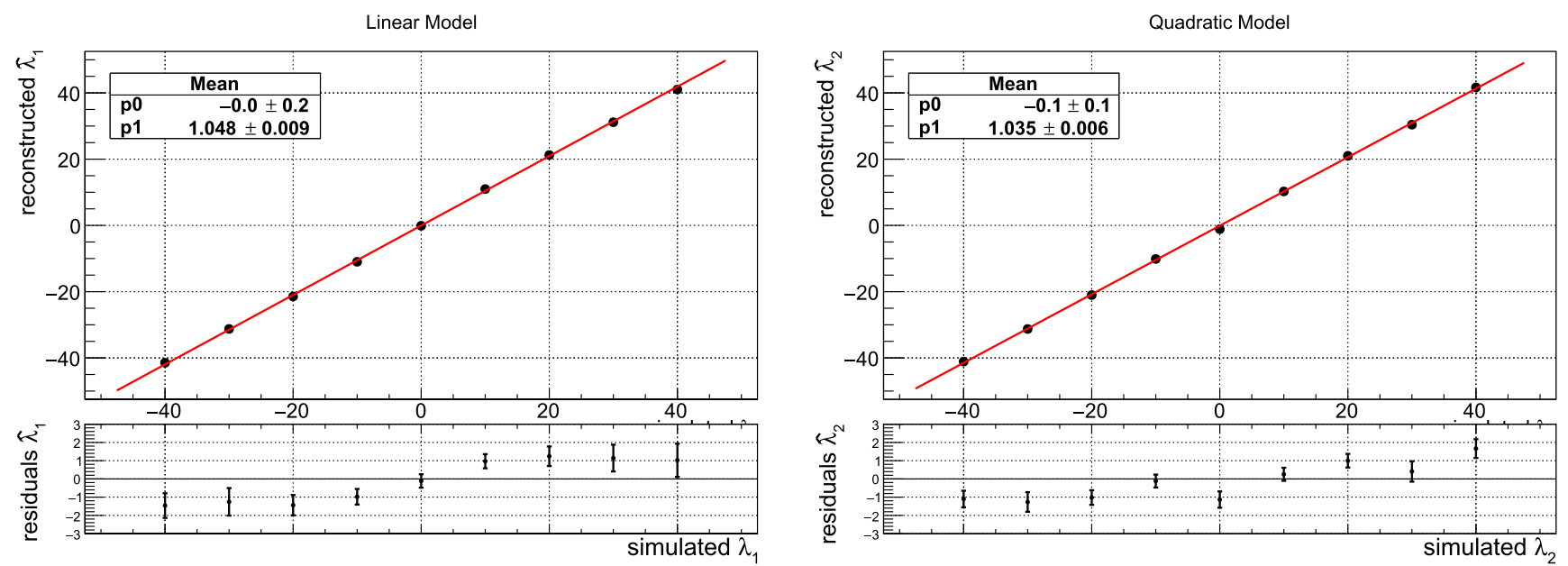

Figure 4. Reconstructed LIV parameter distributions $\widehat{\lambda}$ as a function of simulated intensities $\lambda$. The mean values have been fitted to a linear response function, with the fit results shown in the inserted box. Below the fit, residuals are shown.

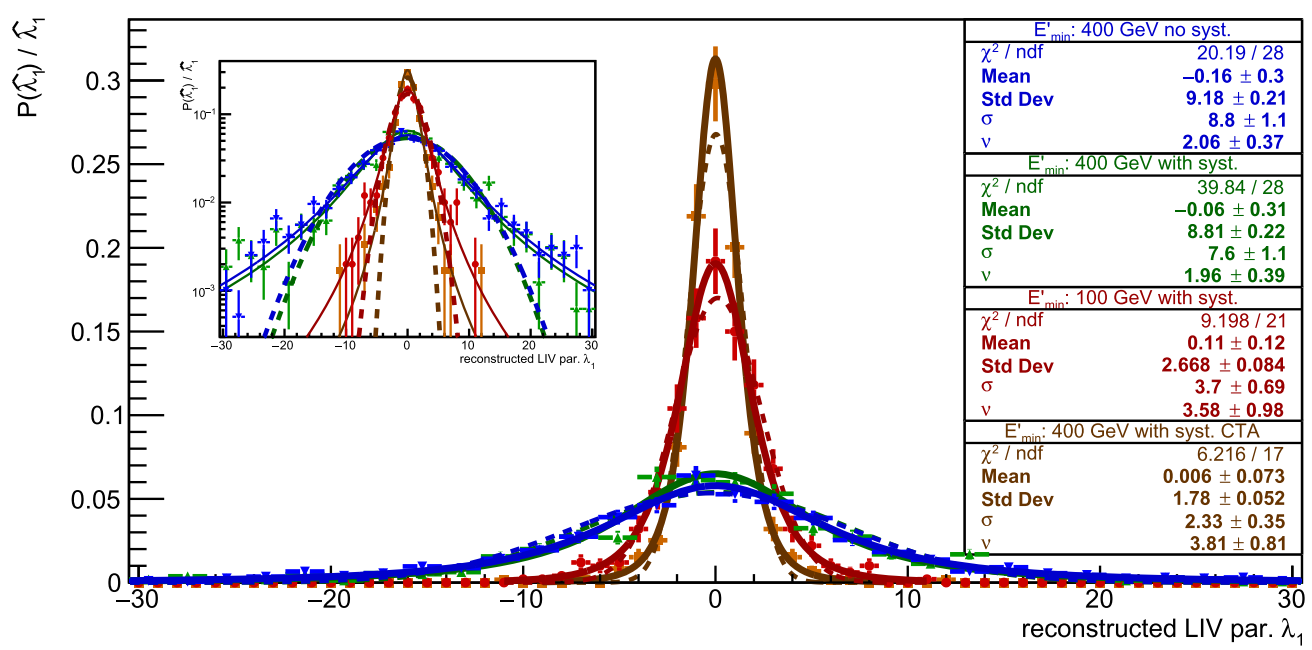

Figure 5. Distribution of estimated linear LIV intensities $\hat{\lambda}_{1}$ for $1000 \mathrm{MC}$ simulations with no LIV each, for four example cases: $E_{\min }^{\prime}=400 \mathrm{GeV}$ and no additional systematics simulated (blue); $E_{\min }^{\prime}=400 \mathrm{GeV}$ and additional systematics simulated on the absolute energy and flux scales (green); $E_{\min }^{\prime}=100 \mathrm{GeV}$ with the same systematics simulated (red); and $E_{\min }^{\prime}=400 \mathrm{GeV}$, with statistics 10 times higher on signal and background, with energy resolution and systematics simulated for the case of CTA (orange). All curves are fit to a normal function (dashed lines) and a Student's $t$-distribution (full lines). All fit results are shown in the Table 4. The inlet shows the same figure in logarithmic scale, to make the tails of the distributions better visible.

emphasize, however, that the measured absence of any linear or quadratic energy dependency of the mean pulse position (up to our sensitivity) makes rather unlikely any intrinsic effect of the same size and opposite direction, canceling LIV effects.

\section{Results and Discussion}

The 95\% CL limits obtained with the calibrated profiled likelihood method are shown in Table 6, with and without including systematic uncertainties. One can see that the profile likelihood method improves the limits by about a factor of four to five with respect to the simple peak search algorithm (shown in Table 2), if the same energy range is used. This is expected because the test statistic Equation (8) exploits additional information, like the constancy of the signal over time (through the condition that the expected number of events for the different observation periods is proportional to the respective observation time), the characteristics of the fluctuation of the expected signal, and last but not least, the continuous linear (or quadratic) evolution of the signal with energy. Along with -and necessary for-such an improvement is the stronger confinement of the nuisance parameters, particularly the mean pulse position $\phi_{\mathrm{P} 2}$, which strongly correlates with the LIV scale (correlation coefficient $\rho \sim 0.5$ ).

Our new limits improve previous constraints from the Crab Pulsar (Otte 2011) by almost a factor of three for the linear case, and by about an order of magnitude for the quadratic case (depending on how systematic uncertainties, not mentioned in Otte (2011), are accounted). For the linear case, our limits are still two orders of magnitude below the best experimental results obtained from GRB090510 (Vasileiou et al. 2013) (see Table 6). For the quadratic case, however, the current best constraints from Vasileiou et al. (2013) are only about a factor of two better than our limits. It should be noted, however, that the limit of $E_{\mathrm{QG}_{2}}>1.3 \times 10^{11} \mathrm{GeV}$ reported in their abstract does not incorporate the additional systematic uncertainty of $10 \%$ from instrumental effects, estimated in their Section 6B. Moreover, that limit applies only to the subluminal case (the superluminal limit from the same method is a factor seven worse) and came out as the best of three statistical methods applied. If we consider their 


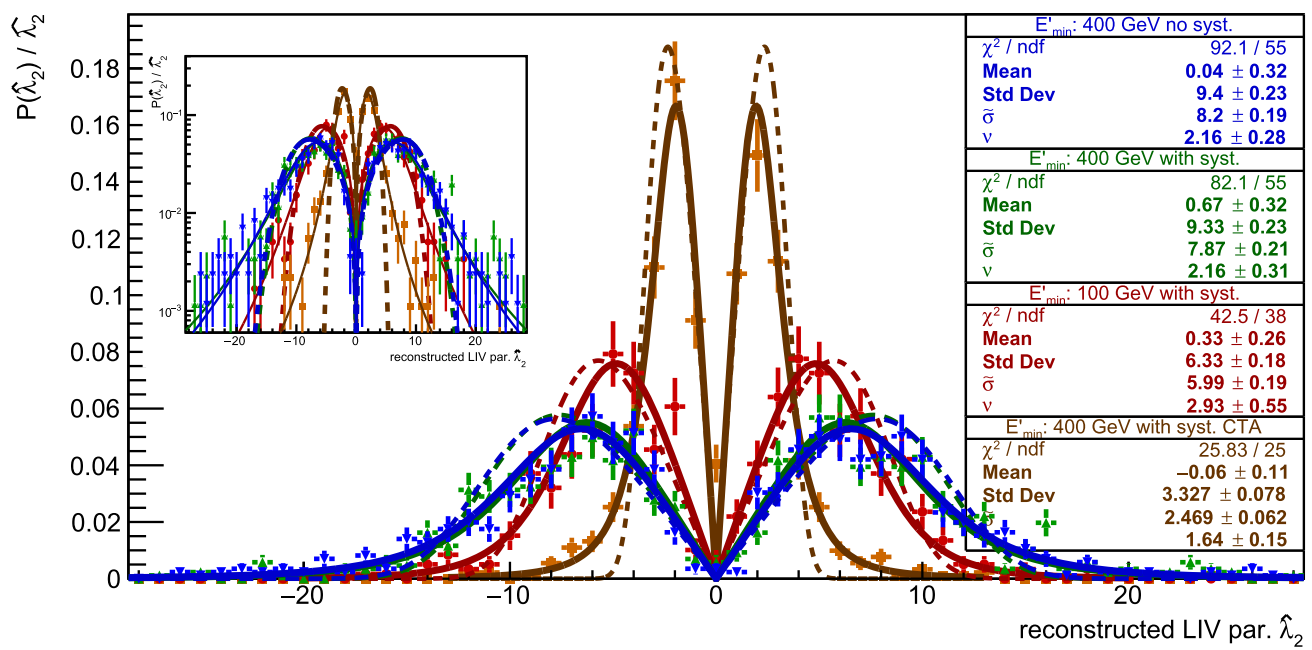

Figure 6. Distribution of estimated quadratic LIV intensities $\widehat{\lambda}_{2}$ for $1000 \mathrm{MC}$ simulations with no LIV each, for four exemplary cases: $E_{\min }^{\prime}=400 \mathrm{GeV}$ and no additional systematics simulated (blue), $E_{\min }^{\prime}=400 \mathrm{GeV}$ and additional systematics simulated on the absolute energy and flux scales (green), $E_{\min }^{\prime}=400 \mathrm{GeV}$, $E_{\min }^{\prime}=150 \mathrm{GeV}$ with the same systematics simulated, and a ten times higher statistics on signal and background, with systematics on the absolute energy and scale simulated for the case of CTA (orange). All curves are fit to a converted normal function (Equation (19), dashed lines) and a converted Student's $t$-distribution (Equation (20), full lines). The fit results of the latter are shown in the text boxes.

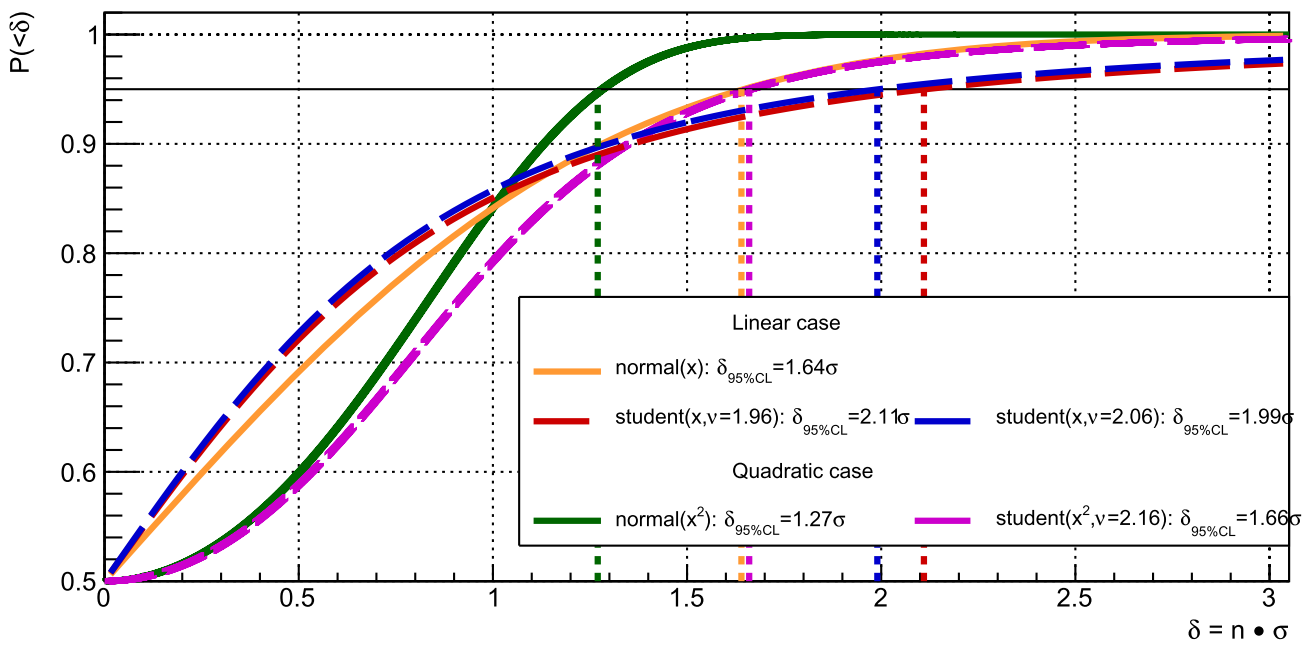

Figure 7. Ninety-five percent confidence limits for the different used distributions.

likelihood results only, and include the mentioned systematic uncertainty, our limits are only $30 \%$ and $60 \%$ lower for the subluminal and superluminal cases, respectively. Even better, though rather model-dependent, constraints on subluminal quadratic LIV have been recently published by Rubstov et al. (2017) and Martínez-Huerta \& Pérez-Lorenzana (2017), using the highest energy photons observed from the Crab Nebula, and the apparent absence of a modification of the Bethe-Heitler cross-section for pair production in the atmosphere, or the absence of photon decay at these energies. A detailed treatment of the systematics inherent to the IACT technique, and in the source spectra themselves, is, however, still missing in both analyses.

Given the strong arguments for an experimental exclusion of any linear LIV effects (Götz et al. 2014; Kislat \& Krawczynski 2017), limits constraining the quadratic dispersion of photons with energy have now become of greater interest. Moreover, if LIV is not isotropic, 25 non-birefringent coefficients must be constrained via direction dependent limits (Kislat \& Krawczynski 2015) whose current best values are five to six orders of magnitude worse.
Unlike flaring astrophysical sources like AGNs or GRBs, pulsar data can be continuously accumulated and statistics improved thereby. The likelihood is currently still dominated by background fluctuations, as well as un-resolved systematics, particularly the pulse shape and its evolution with energy. Such effects have possibly been found between 200 and $300 \mathrm{GeV}$, although the given statistics does not allow to claim firm detection. More data will eventually allow to shed light on the pulse evolution in this energy range and subsequently include events with reconstructed energies below $400 \mathrm{GeV}$ into the likelihood analysis. Our simulations (see Figure 8) have shown that this possibility alone may already improve the limits by at least a factor of two. Moreover, more data will allow to better model the pulse shape itself and take less conservative choices than the used Gaussian pulse shape with fixed symmetric width.

The possibility to take regular data on the Crab Pulsar with a telescope system at the zenith of its performance (Aleksić et al. 2016b) will now permit to regularly improve the sensitivity, and even plan such observations based on numerical predictions of 

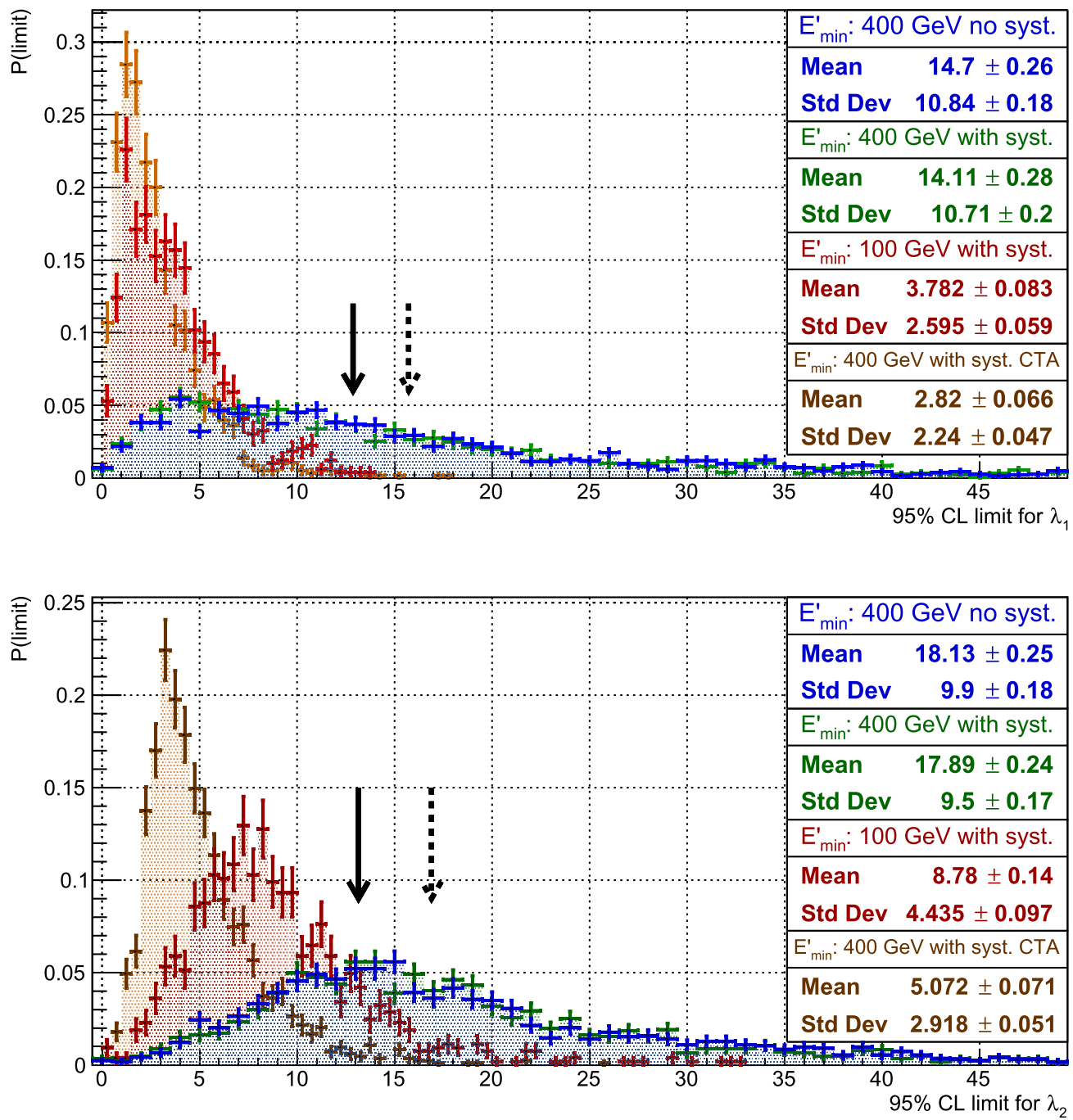

Figure 8. Distribution of 95\% confidence limits for $\lambda_{1}$ (top) and $\lambda_{2}$ (bottom) for the four simulated case scenarios. The arrows denote the experimentally found limits (full line for subluminal behavior, dashed lines for superluminal scenarios).

such improvements. A data set of $2000 \mathrm{hr}$ of stereo data, something perfectly within reach for the MAGIC collaboration, given the regular observation of the Crab Pulsar for calibration purposes, can hence ensure an improvement of the quadratic limit by a factor of two, using data above $400 \mathrm{GeV}$ reconstructed energy alone. Within a framework of collaboration between the different current IACT installations, a significantly higher amount of data is even plausible. Such a limit will reach the current world-best constraints, but has the possibility to go well beyond these, because these data can also help to better understand pulse evolution of the inter-pulse, and such would allow to include events below $400 \mathrm{GeV}$ in the likelihood.

Moreover, it is hoped that the Gaia mission (Gaia Collaboration et al. 2016) will soon be able to measure the distance to Crab to at least an order of magnitude better precision, removing one of the main uncertainties to these limits.

\section{Summary and Conclusions}

We have made use of the profile likelihood method and the Crab Pulsar signal above $400 \mathrm{GeV}$ detected by the MAGIC gamma-ray telescopes (Ansoldi et al. 2016) to perform a test on LIV involving an additional linear or quadratic dispersion relation term with energy for photons. No significant correlation between arrival time and energy of the pulsar photons is observed, and upper limits on the linear and quadratic energy scale of LIV have been derived. The profile likelihood has been carefully calibrated with respect to its bias and coverage properties. For the first time for the Crab Pulsar, systematic uncertainties have been studied and included in the limits, apart from overall conservative choices in the selection of pulse shape models and tested energy ranges.

While the obtained limits are less constraining for the linear case, they come to lie at less than a factor two from the current best limit from GRBs for the interesting quadratic case (Vasileiou et al. 2013), depending on which of the several limits in Vasileiou et al. (2013) are chosen. There is nevertheless a large potential for improvement, once the form of the pulse shapes and their evolution with energy are better understood. We observe hints for such an evolution, particularly in the range between 200 and $300 \mathrm{GeV}$, although statistics do not allow yet to make a firm claim.

This paper brings back pulsars to the class of astrophysical objects that are useful (and competitive) to investigate time-offlight differences of energetic photons. Due to the stable and continuous nature of pulsar emission, limits can now be constantly improved over time, and corresponding observations planned accordingly. Source intrinsic effects that might mimic a possible LIV signal from flaring sources (see, e.g., Bednarek \& 


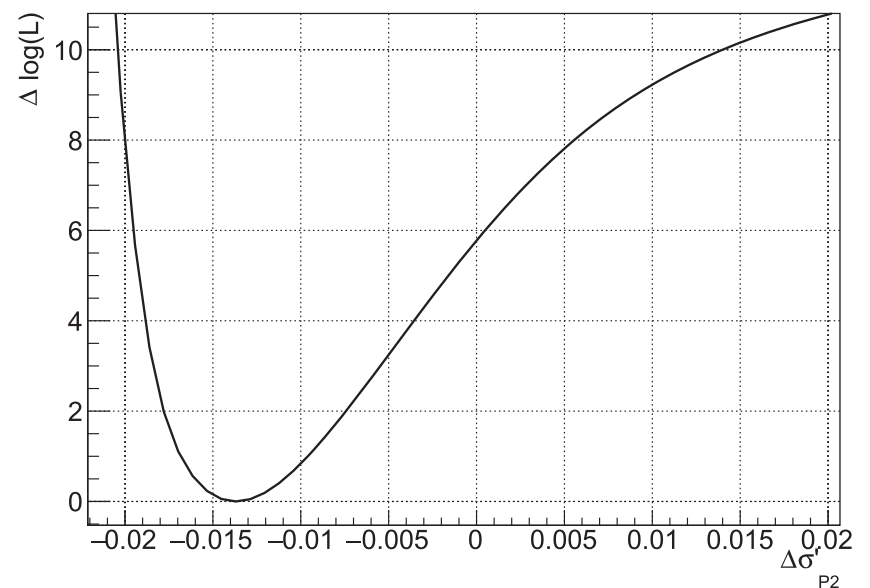

Figure 9. Log-likelihood ratio as a function of the asymmetry parameter $\Delta \sigma_{\mathrm{P} 2}^{\prime}$.

Table 5

List of Studied Systematic Uncertainties

\begin{tabular}{lcc}
\hline \hline Systematic Effect & Size $\left(E_{\mathrm{QG}_{1}}\right)$ & Size $\left(E_{\mathrm{QG}_{2}}\right)$ \\
\hline Background estimation & $<10 \%$ & $<15 \%$ \\
Absolute energy and flux scale & $<7 \%$ & $<5 \%$ \\
Different pulse shapes & $<6 \%$ & $0 \%$ \\
Cutoff in energy spectrum & $<30 \%$ & $<30 \%$ \\
Contribution from the bridge & $<5 \%$ & $<5 \%$ \\
Distance Crab Pulsar & $<25 \%$ & $<12 \%$ \\
\hline Total & $<42 \%$ & $<36 \%$ \\
\hline
\end{tabular}

Note. The effects labeled with an asterisk have been found to improve only the limits.

Table 6

95\% CL Limits from the Profile Likelihood Method and the Best Limits from GRB090510 Obtained by Vasileiou et al. (2013)

\begin{tabular}{|c|c|c|c|c|}
\hline \multirow{2}{*}{ Case } & \multicolumn{2}{|c|}{ Crab Pulsar (This Paper) } & \multicolumn{2}{|c|}{ GRB090510 } \\
\hline & $\begin{array}{c}(\mathrm{W} / \mathrm{o} \\
\text { Systematics })\end{array}$ & $\begin{array}{c}\text { (Incl. } \\
\text { Systematics) }\end{array}$ & $\begin{array}{l}\text { (Best of } 3 \\
\text { Methods) }\end{array}$ & (Likelihood) \\
\hline \multicolumn{5}{|c|}{$E_{\mathrm{QG}_{1}}(\mathrm{GeV})$} \\
\hline$\xi_{1}=+1$ & $7.8 \times 10^{17}$ & $5.5 \times 10^{17}$ & $9.3 \times 10^{19}$ & $6.3 \times 10^{19}$ \\
\hline$\xi_{1}=-1$ & $6.4 \times 10^{17}$ & $4.5 \times 10^{17}$ & $1.3 \times 10^{20}$ & $1.3 \times 10^{20}$ \\
\hline \multicolumn{5}{|c|}{$E_{\mathrm{QG}_{2}}(\mathrm{GeV})$} \\
\hline$\xi_{2}=+1$ & $8.0 \times 10^{10}$ & $5.9 \times 10^{10}$ & $1.3 \times 10^{11}$ & $8.6 \times 10^{10}$ \\
\hline$\xi_{2}=-1$ & $7.2 \times 10^{10}$ & $5.3 \times 10^{10}$ & $9.4 \times 10^{10}$ & $9.4 \times 10^{10}$ \\
\hline
\end{tabular}

Note.

${ }^{a} 10 \%$ systematic uncertainty due to instrumental effects not included.

Wagner 2008; Zheng \& Zhang 2011) are thus being diversified, adding to the robustness of obtained limits and/or possible future signals.

A combination of the profile likelihood obtained in this paper, with those from similar searches using other sources and instruments, like the strong AGN flares observed by MAGIC and H.E.S.S. (Albert et al. 2008; Abramowski et al. 2011), and combinations of GRBs (Vasileiou et al. 2013) can be another promising way to further constrain the effects of LIV, particularly with concern to the quadratic energy dependency of the photon time of flight. The arrival of the next-generation VHE gamma-ray observatory, the Cherenkov Telescope Array (CTA), will easily improve this limit even if a factor of ten less observation time is dedicated to the Crab pulsar.

We would like to thank the Instituto de Astrofísica de Canarias for the excellent working conditions at the Observatorio del Roque de los Muchachos in La Palma. The financial support of the German BMBF and MPG, the Italian INFN and INAF, the Swiss National Fund SNF, the ERDF under the Spanish MINECO (FPA2015-69818-P, FPA2012-36668, FPA2015-68378-P, FPA2015-69210-C6-2-R， FPA201569210-C6-4-R, FPA2015-69210-C6-6-R, AYA2015-71042-P, AYA2016-76012-C3-1-P, ESP2015-71662-C2-2-P, CSD200900064), and the Japanese JSPS and MEXT is gratefully acknowledged. This work was also supported by the Spanish Centro de Excelencia "Severo Ochoa" SEV-2012-0234 and SEV-2015-0548, and Unidad de Excelencia "María de Maeztu" MDM-2014-0369, by the Croatian Science Foundation (HrZZ) Project 09/176, the University of Rijeka Project 13.12.1.3.02, the DFG Collaborative Research Centers SFB823/C4 and SFB876/C3, and by the Polish MNiSzW grant 2016/22/M/ ST9/00382.

\section{Appendix A Used Data Samples}

Because the energy reconstruction and effective collection area of the system were different for each combination of camera hardware (see, e.g., Figure 1 of Ansoldi et al. 2016), trigger, and readout system, the data had to be divided in several subsamples, each with similar instrumental response. This data set was down-selected to more than $300 \mathrm{hr}$ of excellent quality data, including particularly medium and highzenith angle observations that provide better sensitivity above about $800 \mathrm{GeV}$ (Aleksić et al. 2016b).

Table 7 lists the resulting 19 data samples used for this study. The first two sets (I and II) were taken with one telescope in stand-alone mode (see Aliu et al. 2009) and had an energy resolution of around $20 \%$ in the energy range from $400 \mathrm{GeV}$ to $1 \mathrm{TeV}$. The rest of the data samples were taken with two telescopes, operated as a stereoscopic system (see Aleksić et al. $2012 b$ ). These have an energy resolution of $15-17 \%$ at $\sim 1 \mathrm{TeV}$ (Aleksić et al. 2016b). Major upgrades were carried out in mid-2012 and mid-2013, first replacing the readout system, and in the next year, the camera of the first telescope, to achieve a system with almost identical telescopes. The data samples III-VI were taken before the major upgrade (Aleksić et al. 2016a), while samples VII-XIX are from after the upgrade (Aleksić et al. 2016b). From 2012 on, an upgraded sumtrigger (García et al. 2014) was tested on Crab, together with the normal coincidence trigger, and was used to reduce the effective energy threshold of the system (Aliu et al. 2008). However, those events triggered by the sum trigger, and not the standard coincidence trigger, were not included in this analysis.

Using the ephemeris provided by the Jodrell Bank Observatory (Lyne \& Roberts 2014), a phase value was assigned to each of the recorded events with an accuracy of about $4 \mu \mathrm{s}$ (see Section 4.6 of Garrido 2015) using the TEMPO2 package (Hobbs et al. 2006) (and cross-checked by our own code (López Moya 2006)). 

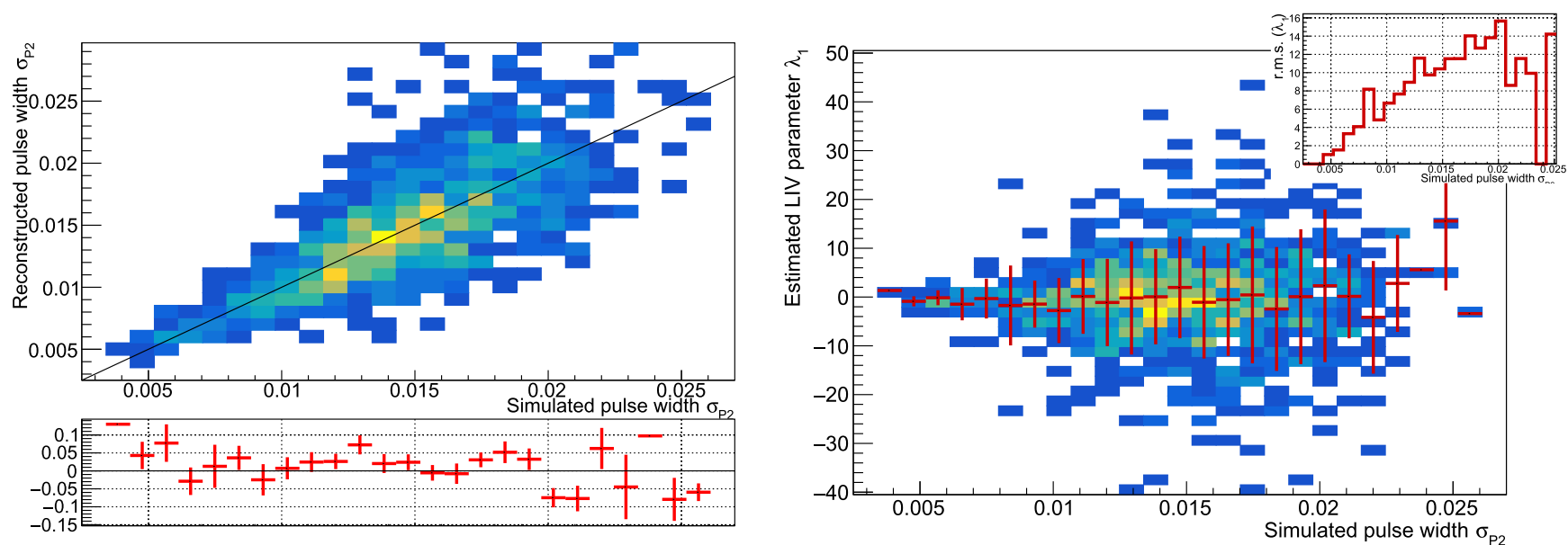

Figure 10. Left: distribution of reconstructed pulse width $\widehat{\sigma}_{\mathrm{P} 2}$ vs. simulated pulse width $\sigma_{\mathrm{P} 2}$, for a set of $1000 \mathrm{MC}$ simulations involving variation of all nuisance parameters, under absence of LIV. Below, the residuals are shown. Right: distribution of reconstructed linear LIV intensities $\widehat{\lambda}_{1}$ for the same set of simulations, as a function of the simulated pulse width $\sigma_{\mathrm{P} 2}$. The inlet shows the rms of $\widehat{\lambda}_{1}$.

Table 7

Summary of the Used Data Samples

\begin{tabular}{|c|c|c|c|c|c|}
\hline Data Set & $\begin{array}{c}\text { Observation } \\
\text { Cycles }\end{array}$ & $\begin{array}{c}\text { Zenith Angle } \\
\text { Range } \\
\text { (deg.) }\end{array}$ & $\begin{array}{l}\text { Effective } \\
\text { On-time } \\
\text { (hr) }\end{array}$ & $\begin{array}{l}\text { Telescope } \\
\text { System }\end{array}$ & $\begin{array}{l}\text { Observation } \\
\text { Configuration }\end{array}$ \\
\hline I & $2-4$ & $5-35$ & 31 & mono & wobble \\
\hline III & $5-6$ & $5-35$ & 40 & stereo & wobble \\
\hline IV & $5-6$ & $35-50$ & 16 & stereo & wobble \\
\hline V & $5-6$ & $50-62$ & 5 & stereo & wobble \\
\hline VIII & 7 & $35-50$ & 2 & stereo & sumtrigger \\
\hline IX & 7 & $5-35$ & 5 & stereo & sumtrigger \\
\hline $\mathrm{X}$ & 7 & $35-50$ & 8 & stereo & sumtrigger \\
\hline XI & 8 & $5-35$ & 22 & stereo & sumtrigger \\
\hline XII & 8 & $35-50$ & 5 & stereo & sumtrigger \\
\hline XIII & 8 & $50-70$ & 12 & stereo & sumtrigger \\
\hline XVIII & 9 & $35-50$ & 6 & stereo & wobble \\
\hline XIX & 9 & $50-70$ & 8 & stereo & wobble \\
\hline
\end{tabular}

Note. Observation cycles are MAGIC-internal numbers where each cycle corresponds to roughly one year. The telescopes were operating first with only one telescope (in mono-mode), but later a second telescope was added and stereo observations made possible. Observations can either be carried out in wobble mode (Fomin et al. 1994), or on mode, where the source is imaged on to the center of the camera. Data sets labeled sumtrigger contained additional events triggered by the sum trigger (García et al. 2014).

\section{Appendix B \\ Dependency of the Spread of Estimated LIV Parameters on Nuisance Parameters}

The effect of the variations of the nuisance parameter $\sigma_{\mathrm{P} 2}$ the spread of $\widehat{\lambda}_{1}$ is displayed in Figure 10. One can see that the nuisance parameter is reconstructed correctly on average, i.e., it shows no bias with respect to the simulated ones. The reconstructed LIV parameter $\widehat{\lambda}_{1}$, although not correlating with the simulated nuisance parameter, shows a reconstruction uncertainty that increases with larger values of simulated $\sigma_{\mathrm{P} 2}$. This behavior ultimately produces a stronger peaked distribution of $\widehat{\lambda}_{1}$ with wider tails.

\section{Appendix C \\ Exponential Cutoff Limit}

We define a test statistic $D$ for $E_{b}$, based on the profile likelihood method, similar to the one defined in Equation (7), and set the LIV parameters $\lambda_{1,2}$ to zero:

$$
D\left(E_{b} \mid \boldsymbol{X}\right)=-2 \ln \left(\frac{\mathcal{L}\left(E_{b} ; \widehat{\boldsymbol{\nu}} \mid \boldsymbol{X}, \lambda_{1,2}=0\right)}{\mathcal{L}\left(\widehat{E}_{b} ; \widehat{\boldsymbol{\nu}} \mid \boldsymbol{X}, \lambda_{1,2}=0\right)}\right) .
$$

A minimum of $D$ is then found for no cutoff, i.e., $E_{b}=\infty$. Figure 11 shows the test statistic as a function of $E_{b}$. A $95 \%$ CL limit can be evaluated at that value of $E_{b}$ where $D=2.71$, 


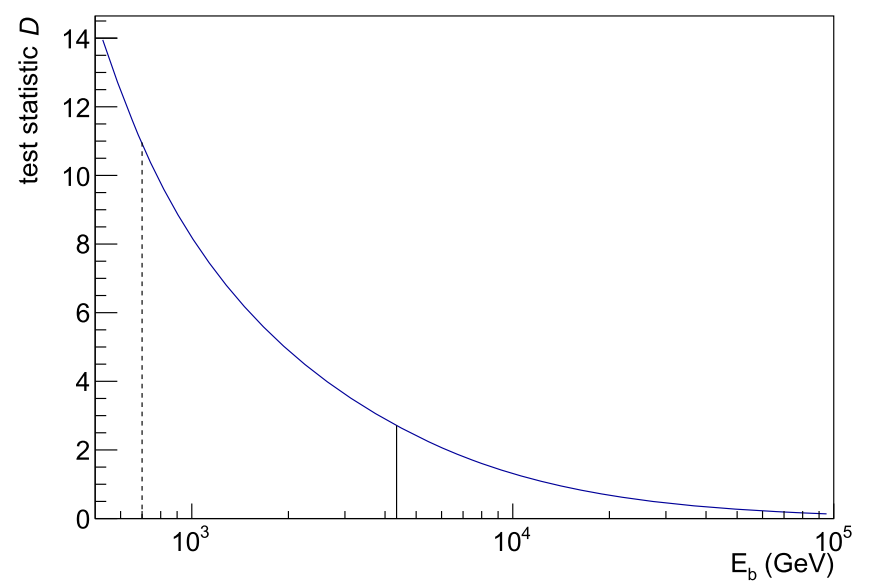

Figure 11. Test statistic (Equation (23)) as a function of the exponential cutoff energy $E_{b}$. The full line indicates the energy where $D=2.71$; the dashed line shows the previous limit $E_{b}=700 \mathrm{GeV}$.

resulting in $E_{b} \gtrsim 4.3 \mathrm{TeV}$. The value of $D$ is found to be 11.0 at the previous limit $E_{b}>700 \mathrm{GeV}(95 \% \mathrm{CL})$ published in Ansoldi et al. (2016), who used a binned likelihood approach with fixed nuisance parameters $\phi_{\mathrm{P} 2}$ and $\sigma_{\mathrm{P} 2}$.

\section{ORCID iDs}

J. Becerra González (i) https://orcid.org/0000-0002-6729-9022 W. Bednarek (i) https://orcid.org/0000-0003-0605-108X

S. Covino (1) https://orcid.org/0000-0001-9078-5507

A. Domínguez (ํ) https://orcid.org/0000-0002-3433-4610

M. Gaug (i) https://orcid.org/0000-0001-8442-7877

M. Hayashida (i) https://orcid.org/0000-0003-2252-3411

H. Kubo (1) https://orcid.org/0000-0001-9159-9853

A. Niedzwiecki (1) https://orcid.org/0000-0002-8541-8849

M. Nievas Rosillo (1) https://orcid.org/0000-0002-8321-9168

K. Nilsson (i) https://orcid.org/0000-0002-1445-8683

S. Paiano 1 https://orcid.org/0000-0002-2239-3373

J. M. Paredes (1) https://orcid.org/0000-0002-1566-9044

M. Ribó i https://orcid.org/0000-0002-9931-4557

F. Tavecchio (1) https://orcid.org/0000-0003-0256-0995

A. Treves (ㄴ) https://orcid.org/0000-0002-0653-6207

\section{References}

Abdo, A., Ackermann, M., Ajello, M., et al. 2010a, ApJ, 708, 1254 Abdo, A., Ajello, M., Allafort, A., et al. 2013, ApJS, 208, 13 Abdo, A. A., Acerkmann, Ajello, M., et al. 2010b, ApJ, 708, 1310 Abramowski, A., Acero, F., Aharonian, F., et al. 2011, APh, 34, 738 Actis, M., Agnetta, G., Aharonian, F., et al. 2011, ExA, 32, 193 Aharonian, F. A., Bogovalov, S. V., \& Khangulyan, D. 2012, Natur, 482, 507 Albert, J., Aliu, E., Anderhub, H., et al. 2008, PhLB, 668, 253 Aleksić, J., Alvarez, E. A., Antonelli, L. A., et al. 2011, ApJ, 742, 43 Aleksić, J., Alvarez, E., Antonelli, L. A., et al. 2012a, A\&A, 540, A69 Aleksić, J., Alvarez, E. A., Antonelli, L. A., et al. 2012b, APh, 35, 435 Aleksić, J., Ansoldi, S., Antonelli, L. A., et al. 2014a, A\&A, 565, L12 Aleksić, J., Ansoldi, S., Antonelli, L. A., et al. 2014b, JCAP, 2, 008 Aleksić, J., Ansoldi, S., Antonelli, L. A., et al. 2016a, APh, 72, 61 Aleksić, J., Ansoldi, S., Antonelli, L. A., et al. 2016b, APh, 72, 76 Aliu, E., Anderhub, H., Antonelli, L. A., et al. 2008, Sci, 322, 1221 Aliu, E., Anderhub, H., Antonelli, L. A., et al. 2009, APh, 30, 293 Aliu, E., Arlen, T., Aune, T., et al. 2011, Sci, 334, 69

Amelino-Camelia, G., Ellis, J., Mavromatos, N. E., Nanopoulos, D. V., \& Sarkar, S. 1998, Natur, 393, 763
Amelino-Camelia, G., \& Smolin, L. 2009, PhRvD, 80, 084017

Ansoldi, S., Antonelli, L. A., Antoranz, P., et al. 2016, A\&A, 585, A133

Barlow, R. 1990, NIMPA, 297, 496

Bednarek, W., \& Wagner, R. M. 2008, A\&A, 486, 679

Bernlöhr, K., Barnacka, A., Becherini, Y., et al. 2013, APh, 43, 171

Blobel, V., \& Lohmann, E. 2012, Statistische und Numerische Methoden der Datenanalyse (Leipzig: Verlag Teubner Stuttgart)

Bogovalov, S. V. 2014, MNRAS, 443, 2197

Brun, R., \& James, F. 2015, TMinuit Class Reference, https://root.cern.ch/ root/html534/TMinuit.html

Brun, R., \& Rademakers, F. 1996, NIMPA, 389, 81

Bühler, R., \& Blandford, R. 2014, RPPh, 77, 066901

Burgess, C. P., Cline, J. M., Filotas, E., Matias, J., \& Moore, G. D. 2002, JHEP, 3, 043

Colladay, D., \& Kostelecký, V. A. 1998, PhRvD, 58, 116002

Cortina, J., Goebel, F., Schweizer, T. \& For the MAGIC Collaboration 2009, arXiv:0907.1211

Covino, S., \& Gotz, D. 2016, A\&AT, 29, 205

Cowan, G., Cranmer, K., Gross, E., \& Vitells, O. 2011, EPJC, 71, 1554

Domínguez, A., \& Ajello, M. 2015, ApJL, 813, L34

Douglas, M. R., \& Nekrasov, N. A. 2001, RvMP, 73, 977

Du, Y. J., Qiao, G. J., \& Wang, W. 2012, ApJ, 748, 84

Fierro, J. M., Michelson, P. F., Nolan, P. L., \& Thompson, D. J. 1998, ApJ, 494, 734

Fomin, V. P., Stepanian, A. A., Lamb, R. C., et al. 1994, APh, 2, 137

Gaia Collaboration, Prusti, T., De Bruine, J. H., et al. 2016, A\&A, 595, A1

Gambini, R., \& Pullin, J. 1999, PhRvD, 59, 124021

García, J. R., Dazzi, F., Häfner, D., et al. 2014, arXiv:1404.4219

Garrido, D. 2015, PhD thesis, Universitat Autònoma de Barcelona

Gaug, M., Berge, D., Daniel, M., et al. 2014, Proc. SPIE, 9149, 19

Götz, D., Laurent, P., Antier, S., et al. 2014, MNRAS, 444, 2776

Gubitosi, G., Pagano, L., Amelino-Camelia, G., Melchiorri, A., \& Cooray, A. 2009, JCAP, 8, 021

Hamed-Arkani, N., Cheng, H. S., Luty, M. A., \& Mukohyama, S. 2004, JHEP, 5,074

Hirotani, K. 2001, ApJ, 549, 495

Hirotani, K. 2013, ApJ, 766, 98

Hobbs, G. B., Edwards, R. T., \& Manchester, R. N. 2006, MNRAS, 369, 655

Hořava, P. 2009, PhRvD, 79, 084008

James, F., \& Roos, M. 1975, CoPhC, 10, 343

Kaaret, P. 1999, A\&A, 345, L32

Kaplan, D. L., Chatterjee, S., Gaensler, B. M., \& Anderson, J. 2008, ApJ, 677, 1201

Kislat, F., \& Krawczynski, H. 2015, PhRvD, 92, 045016

Kislat, F., \& Krawczynski, H. 2017, PhRvD, 95, 083013

Kostelecký, V. A. 2004, PhRvD, 69, 105009

Kostelecký, V. A., \& Mewes, M. 2008, ApJL, 689, L1

Kostelecký, V. A., \& Samuel, S. 1989, PhRvD, 39, 683

Kranmer, K. 2015, arXiv:1503.07622

López Moya, M. 2006, PhD thesis, Universidad Complutense de Madrid

Lyne, A., \& Roberts, M. 2014, Jodrell Bank Crab Pulsar Monthly Ephemeris, http://www.jb.man.ac.uk/ pulsar/crab.html

Lyutikov, M., Otte, N., \& McCann, A. 2012, ApJ, 754, 33

Magueijo, J., \& Smolin, L. 2002, PhRvL, 88, 190403

Martínez-Huerta, H., \& Pérez-Lorenzana, A. 2017, PhRvD, 95, 63001

Martinez, M., \& Errando, M. 2009, APh, 31, 226

Massaro, E., Campana, R., Cusumano, G., \& Mineo, T. 2006, A\&A, 459, 859

Massaro, E., Cusumano, G., Litterio, M., \& Mineo, T. 2000, A\&A, 361, 695

Mattingly, D. 2005, LRR, 8, 5

Mineo, T., Cusumano, G., Segreto, A., et al. 1997, A\&A, 327, L21

Murphy, S. A., \& van der Vaart, A. W. 2000, J. Am. Stat. Assoc., 95, 449

Olive, K. \& Particle Data Group 2014, ChPhC, 38, 090001

Otte, N. 2011, ICRC, 7, 256

Rovelli, C. 2004, Quantum Gravity (Cambridge: Cambridge Univ. Press)

Rubstov, G., Satunin, P., \& Sibiryakov, S. 2017, Journal of Cosmology and Astroparticle Physics, 5, 49

Trimble, V. 1973, PASP, 85, 579

Vasileiou, V., Jacholkowska, A., Piron, F., et al. 2013, PhRvD, 87, 122001

Walck, C. 1996, Hand-book on Statistical Distributions for Particle Physicists (2nd ed.; Univ. Stockholm)

Warner, B., \& Nather, R. E. 1969, Natur, 222, 157

Zheng, Y. G., \& Zhang, L. 2011, ApJ, 728, 105 